\title{
A splitting theorem for nonnegatively curved Alexandrov spaces
}

\author{
ANDREAS WÖRNER
}

\begin{abstract}
We study Alexandrov spaces of nonnegative curvature whose boundaries consist of several strata of codimension 1. If the space is compact and the common intersection of all boundary strata is empty, then the space is a metric product. In particular, this is fulfilled if the compact space has dimension $n$ and contains more than $n+1$ boundary strata. The splitting factors are in general non-flat.
\end{abstract}

$53 \mathrm{C} 23 ; 51 \mathrm{H} 25$

\section{Introduction}

Many of the known splitting theorems for nonnegatively curved Riemannian manifolds provide flat factors. For instance, according to Toponogov's Splitting Theorem [28] and its generalization by Cheeger and Gromoll [4], the manifold splits off an $\mathbb{R}$-factor if it contains a straight line. The analogous result for locally compact Alexandrov spaces was proved by Milka [12] (in Russian; a proof in English can be found in the book by Burago-Burago-Ivanov [2]). Later, Mitsuishi [13] eliminated the assumption of local compactness. In finite dimensional Alexandrov spaces the assumptions can be weakened further due to Mashiko [10] and to Alexander-Bishop [1]. They assumed not the existence of a straight line, but of certain non-trivial affine functions.

If one is interested in non-flat factors for a metric splitting, the soul of the space according to Cheeger's and Gromoll's Soul Theorem [5] is a natural candidate. Walshap [29] obtained conditions ensuring that a space splits off its soul. His result has been improved independently by Strake [27] and by Yim [32]. They showed that the splitting happens if, and only if, the normal bundle of the soul has trivial holonomy. Another approach was given by Shioha [26] by considering the ideal boundary: if its radius is close enough to $\pi$, the space splits off its soul. Shioha conjectured that assuming the radius of the ideal boundary to be bigger than $\frac{\pi}{2}$ is already sufficient, which was proved independently by Medonça [11] and by Perelman [19].

A soul theorem for Alexandrov spaces was proved by Perelman in his unpublished preprint [16]. Crucial is his observation that the distance to the boundary is a (strictly) 
concave function if the space has (positive) nonnegative curvature. Hence, the set where the function attains its maximum is a convex subset. If it has boundary, the procedure is iterated. A retraction map is given by the (later in much more generality developed) gradient push or by an iteration of gradient pushes, respectively. It is not known, however, if the obtained Sharafutdinov retraction (compare Sharafutdinov [25]) is a submetry, that is, if it maps balls onto balls preserving their radii. For Riemannian manifolds, in contrast, Perelman proved this fact in his solution of the soul conjecture [18]. The soul conjecture states that if the space has quasi-positive curvature (that is nonnegative curvature and there exists a point of positive curvature), then the soul is a point. For Alexandrov spaces this conjecture is still open.

In this paper we will assume that the boundary of a compact Alexandrov space consists of several components in order to show that the space splits off its soul.

1.1 Definition Let $M$ be a finite dimensional Alexandrov space with lower curvature bound and with boundary $\partial M$. A boundary stratum is an extremal set of locally constant codimension 1 . A collection $F_{1}, \ldots, F_{\ell}$ of boundary strata is a stratification of $\partial M$, if $F_{1} \cup \ldots \cup F_{\ell}=\partial M$ and $\operatorname{codim}\left(F_{i} \cap F_{j}\right) \geq 2 \forall i \neq j$.

For more information about extremal sets and references see Section 2. The boundary $\partial M$ itself is always a boundary stratum according to our definition. If we choose several boundary strata, we always mean distinct elements of some fixed stratification of $\partial M$.

The main result of this paper is the following one.

1.2 Theorem (Splitting Theorem) Let $M$ be an $n$-dimensional compact Alexandrov space of nonnegative curvature with boundary. Assume that $F_{1}, \ldots, F_{\ell+1}$ is a stratification of $\partial M$ and there is $1 \leq k \leq \ell$ such that the following holds.

- $\quad F_{1} \cap \ldots \cap F_{k+1}=\varnothing$,

- $\quad F_{1} \cap \ldots \cap \widehat{F}_{i} \cap \ldots \cap F_{k+1} \neq \varnothing \quad \forall i \in\{1, \ldots, k+1\}$.

(The notation $\widehat{F}_{i}$ means that $F_{i}$ is omitted from the respective collection.)

Then $M$ is isometric to the Euclidean product of nonnegatively curved Alexandrov spaces $S$ and $D$ fulfilling $\operatorname{dim} S=n-k, \operatorname{dim} D=k$, and $S$ can be chosen as any intersection $F_{1} \cap \ldots \cap \widehat{F}_{i} \cap \ldots \cap F_{k+1}$. Then, in addition, the sets $F_{k+2} \cap$ $S, \ldots, F_{\ell+1} \cap S$ form a stratification of $\partial S$, provided $k<\ell$. Otherwise, $S$ has no boundary and is a soul of $M$. 
1.3 Corollary Under the assumptions of Theorem 1.2, the space $M$ has no point of positive curvature.

These assumptions may seem a bit technical. However, if the total number of boundary strata of $M$ is high enough, they are fulfilled automatically.

1.4 Theorem Let $M$ be a nonnegatively curved Alexandrov space with $\partial M \neq \varnothing$. Then the following holds for any subcollection $F_{1}, \ldots, F_{k}$ of any stratification of $\partial M$ : If $k \leq n$ and $F_{1} \cap \ldots \cap F_{k} \neq \varnothing$, then this intersection has locally constant dimension $n-k$. If $k>n$, then $F_{1} \cap \ldots \cap F_{k}$ is always empty. Moreover, for any $k \geq 3$ it is impossible that all intersections of $k-2$ boundary strata $F_{i}$ are nonempty, while all intersections of $k-1$ boundary strata are empty.

1.5 Corollary Under the assumptions of Theorem 1.4, let $M$ be compact and have quasi-positive curvature. Then $F_{1} \cap \ldots \cap F_{k} \neq \varnothing$ if and only if $k \leq n$.

Note that if $M$ has positive curvature, a proof of Corollary 1.5 does not need both, Theorem 1.2 and Theorem 1.4, but follows from the latter one together with Petrunin's generalization [23] of Frankel's Theorem.

Now we may formulate the consequences as follows.

1.6 Corollary If a compact Alexandrov space $M$ of nonnegative curvature and of finite dimension $n$ has more than $n+1$ boundary strata, then it is a metric product.

In case the maximal numbers of boundary strata occur, we get the following results.

1.7 Corollary Let $M$ be a compact $n$-dimensional Alexandrov space of quasipositive curvature. Assume that $M$ contains $n+1$ boundary strata. Then the soul of $M$ is a point. In other words, the soul conjecture holds for Alexandrov spaces with the maximal number of boundary strata.

The subsequent result about spaces of nonnegative curvature has already been proved by Perelman, as we were told. For completeness we also mention it here.

1.8 Corollary The maximal number of boundary strata of a compact $n$-dimensional Alexandrov space $M$ with nonnegative curvature is $2 n$. Equality holds if and only if $M$ is a Euclidean cuboid.

Another consequence of our Splitting Theorem is a weaker version of Shioha's Splitting Theorem, which was mentioned above. Indeed, we get the splitting under stronger assumptions on the radius of the ideal boundary as follows. 


\subsection{Corollary Let $M$ be a noncompact Alexandrov space of nonnegative curvature} without boundary. Assume that the ideal boundary of $M$ has dimension $m$ and it contains $m+2$ points at distance $>\frac{\pi}{2}$ from each other. (In particular, the radius of the ideal boundary is bigger that $\frac{\pi}{2}$.) Then $M$ splits off its soul.

The idea of obtaining structure results by assumptions about boundary decompositions came from Wilking. In [30] he studied orbit spaces of manifolds by Lie group actions. If such a space $M$ has positive curvature in the Alexandrov sense, the number of boundary strata determines the homeomorphism type of $M$ as a stratified space. Namely, if $\operatorname{dim} M=n$ and there are $n+1$ boundary strata, then $M$ is homeomorphic to an $n$-simplex. If there are $k+1<n+1$ boundary strata, then $M$ is homeomorphic to the join of a $k$-simplex and the intersection of all boundary strata. These results carry over to general Alexandrov spaces of positive curvature. Indeed, the main principle of Wilking's proof stays the same if one uses Perelman's Morse theory [17] and his Stability Theorem. All tools needed can be found in papers by Perelman-Petrunin [20] and Kapovitch [7].

This paper is organized as follows. In Section 2 we give a short survey on basic facts about Alexandrov spaces and our notation. In all subsequent sections we always consider Alexandrov spaces of nonnegative curvature. Boundary strata and their distance functions are investigated more closely in Section 3. By Perelman's work, superlevel sets are convex subsets and therefore again Alexandrov spaces. As long as no collapse happens, the boundary strata of such superlevel sets intersect in the same matter as in the original space. This means that we can "shrink" the space by "shifting" boundary strata. In Section 4 we investigate some properties of intersections of boundary strata. In particular, the first part of Theorem 1.4 will be proved. In Section 5 the proof will be completed. We consider the sets where the distance to some boundary stratum is maximal. In other words, we "shrink" our space till it collapses. It will turn out that the resulting space still inherits basic properties of its boundary strata from the original space. More precisely, if the space fulfills the assumptions of the Splitting Theorem, such a maximum set fulfills them, too. The rest of the paper is devoted to the proof of the Splitting Theorem. In Section 6 the basic assumptions will be fixed. Thereafter, in Section 7 we will prove that the space is fibrated into isometric convex subsets. In Section 8 the canonical gradient pushes will turn out to be submetries. In particular, the space can be retracted onto each of those isometric convex subsets. Therefore, these sets are souls. The existence of dual fibrations follows directly, and in Section 9 also some uniqueness will be proved. Namely, the dual fibrations coming from different submetries do in fact coincide. This result enables the proof in Section 10 that all souls are equidistant. At this point, the Splitting Theorem is essentially proved. Remaining details are added in Section 11. 
The results presented here were mostly obtained in the author's $\mathrm{PhD}$ thesis [31]. I am deeply grateful to my advisor Prof. Burkhard Wilking.

\section{Preliminaries and notation}

In this work the term Alexandrov space always means an intrinsic metric space with lower curvature bound in the sense of Burago-Gromov-Perelman [3]. For the basic theory we refer there or to Burago-Burago-Ivanov [2]. Mostly, we consider finite dimensional complete path-connected Alexandrov spaces. The class of such spaces of dimension $n \in \mathbb{N}$ and with curvature bound $\kappa \in \mathbb{R}$ will be denoted by $\operatorname{ALEX}^{n}(\kappa)$. The term dimension always refers to the topological dimension. For Alexandrov spaces, however, it equals the Hausdorff dimension.

For $M \in \operatorname{ALEX}^{n}(\kappa)$ and $p \in M$ the space of directions at $p$ is denoted by $\Sigma_{p}$. It satisfies $\Sigma_{p} \in \operatorname{ALEX}^{n-1}(1)$, and its metric cone $K\left(\Sigma_{p}\right)$ coincides with the tangent cone $T_{p} M$ at $p$. For distinct points $p, q \in M$ a fixed shortest path between $p$ and $q$ is denoted by $p q$. The direction of $p q$ at $p$ is denoted by $\uparrow_{p}^{q}$, while $\uparrow_{p}^{q}$ and $\Uparrow_{p}^{A}$ denote the set of all directions in $\Sigma_{p}$ of shortest paths from $p$ to $q$ or to some closed set $A \subseteq M$, respectively. Distance functions are denoted by $d_{A}:=d(\cdot, A)$.

Let $U \subseteq M$ be an open subset and $f: U \rightarrow \mathbb{R}$ a continuous function. Since for $p \in U$ the tangent cone $T_{p} M$ is the pointed Gromov-Hausdorff limit of blow-ups centered at $p$, this may induce a well-defined function on $T_{p} M$. If this is the case, $f$ is called differentiable at $p$. The differential is a function $d_{p} f: T_{p} M \rightarrow \mathbb{R}$ mapping $v \in T_{p} M$ onto the directional derivative $d_{p} f(v)$. Distance functions are differentiable and, more generally, so is the following class of functions: Let $\lambda \in \mathbb{R}$ and assume that $\partial M=\varnothing$ (otherwise consider the doubling $\bar{M}$, see below). A function $f: U \rightarrow \mathbb{R}$ is called $\lambda$-concave, if $t \mapsto(f \circ \gamma)(t)-\frac{\lambda}{2} t^{2}$ is concave for any shortest path $\gamma$ in $U$. If this holds locally with different values of $\lambda$, then $f$ is called semi-concave. Moreover, semiconcave functions have a well-defined gradient $\nabla_{p} f \in T_{p} M$. It points in the direction where $f$ increases most in first order, and its length satisfies $\left|\nabla_{p} f\right|^{2}=d_{p} f\left(\nabla_{p} f\right)$. This gives rise to well-defined gradient curves and a flow called gradient push. That is a map $\Phi_{f}^{t}: M \rightarrow M$ existing for all time $t \geq 0$. The concept was introduced by Perelman-Petrunin [21]. Our definition is according to Petrunin [24], where detailed information is available. Lytchak [9] investigated gradients in more general spaces.

Further important structure results on Alexandrov spaces were obtained in Perelman's work [17]. He developed a Morse theory in order to show that a finite dimensional Alexandrov space is stratified into topological manifolds. This stratification was adapted 
to the geometry of the space using so-called extremal subsets defined by PerelmanPetrunin [20]. More information and the following equivalent definition can be found in Petrunin's survey paper [24]: For $M \in \operatorname{ALEX}^{n}(\kappa)$ a subset $E \subseteq M$ is extremal if $\Phi_{f}^{t}(E) \subseteq E$ holds for each semi-concave function $f: M \rightarrow \mathbb{R}$ and all times $t \geq 0$. An extremal set is called primitive if it contains no proper extremal subset with nonempty relative interior. A relative version of the Morse Lemma by Perelman and Petrunin shows that also an extremal set is a stratified manifold. It has locally constant dimension if, and only if, it is the union of primitive extremal sets of the same dimension. Therefore, if such a union has codimension 1 , it is a boundary stratum according to our definition.

The boundary $\partial M$ is defined inductively via $p \in \partial M \Longleftrightarrow \partial \Sigma_{p} \neq \varnothing$ (note that a 1-dimensional Alexandrov space is either a line, a circle or an interval). Another characterization of $\partial M$ uses Perelman's Morse theory, which shows that $\partial M$ is the closure of all points $p \in M$ such that $T_{p} M$ is homeomorphic to the half space $\mathbb{R}^{n-1} \times \mathbb{R}_{\geq 0}$. This implies that $\partial M$ is an extremal set. It has codimension 1 and, vice versa, each primitive extremal set of codimension 1 lies in $\partial M$. This follows by induction over dimension and the following important fact about extremal sets: If $E \subseteq M$ is extremal and $p \in E$, then $\Sigma_{p} E$ is extremal in $\Sigma_{p}$. The converse holds if $E$ contains at least two points.

A comprehensive reference for all results related to the Morse Lemma is Kapovitch's paper [7] on Perelman's Stability Theorem, the highlight of structure results in Alexandrov geometry. It states that each compact finite dimensional Alexandrov space $M$ possesses a number $\varepsilon>0$ such that any compact Alexandrov space of the same dimension at Gromov-Hausdorff distance at most $\varepsilon$ is homeomorphic to $M$. Kapovitch extended this result to a relative version using the relative Morse Lemma. Thus, the homeomorphisms from above can be chosen such that they respect the extremal sets of the spaces.

The Gluing Theorem by Petrunin [22] states that two Alexandrov spaces with isometric boundaries can be glued together yielding an Alexandrov space. A special case is the Doubling Theorem by Perelman [16], where two isometric copies of an Alexandrov space $M$ are glued together along $\partial M$. We denote the obtained space by $\bar{M}$. For a subset $A \subseteq M$ let $\bar{A}$ be the preimage of $A$ under the canonical projection $\bar{M} \rightarrow M$.

\section{Boundary strata}

The Doubling Theorem by Perelman also works when gluing along some boundary strata. By this fact, it often suffices to consider spaces without additional boundary strata apart from those of interest. 
3.1 Proposition (Doubling Theorem) Let $M \in \operatorname{ALEX}^{n}(\kappa)$ and let $F$ be a boundary stratum. Then the doubling $\bar{M}$ obtained by gluing along $F$ is an Alexandrov space $\bar{M} \in \operatorname{ALEX}^{n}(\kappa)$. Moreover, if $G$ is another boundary stratum, the set $\bar{G}$ is a boundary stratum of $\bar{M}$.

For the proof just repeat Perelman's one from [16] with $\partial M$ replaced by $F$ and take basic facts about extremal sets into account. This can be found written up in the author's $\mathrm{PhD}$ thesis [31]. As an immediate consequence, also Perelman's concavity result for the distance function $d_{\partial M}$ carries over.

3.2 Corollary Let $M \in \operatorname{ALEX}^{n}(\kappa)$ with $\kappa \geq 0$ and let $F$ be a boundary stratum. Then the distance function $d_{F}$ is concave on $M \backslash F$. It is strictly concave if $\kappa>0$.

Proof If $F \neq \partial M$, consider $\bar{M}$ by gluing along the boundary stratum $\overline{\partial M \backslash F}$ and obtain that $\partial \bar{M}=\bar{F}$. Now use Perelman's result [16, Theorem 6.1] or Petrunin's one [24, Theorem 3.3.1].

3.3 Remark If $M \in \operatorname{ALEX}^{n}(0)$ and $F$ is a boundary stratum, gradient curves of the function $d_{F}$ are also well-defined when starting at any point $p \in F$. This is because $d_{\Sigma_{p} F}$ is strictly concave on $\Sigma_{p} \backslash \Sigma_{p} F$. Thus, there is a unique direction where $d_{F}$ increases most. More details are given in [31, Lemma 2.9].

Note that a gradient curve of $d_{F}$ can leave an extremal set $E$ only if it starts in $E \cap F$. On the other hand, if $E \cap F=\varnothing$ holds, even more can be said.

3.4 Lemma For $M \in \operatorname{ALEX}^{n}(0)$ let $F$ be a boundary stratum. Let $E \subseteq M$ be an extremal set satisfying $E \cap F=\varnothing$. Then $d_{F}$ is constant on $E$, attaining its maximum.

Proof In case $E$ is compact, there are points $p \in E, q \in F$ satisfying $|p q|=|E F| \neq 0$. Assume, by way of contradiction, that there exists $p^{\prime} \in M$ such that $d_{F}\left(p^{\prime}\right)>d_{F}(p)$. By concavity of $d_{F}$ we have that $0<d_{p} d_{F}\left(\uparrow_{p}^{p^{\prime}}\right)=-\cos \left|\uparrow_{p}^{F} \uparrow_{p}^{p^{\prime}}\right|$ and therefore $\measuredangle q p p^{\prime}>\frac{\pi}{2}$. On the other hand, the distance function $\left.d_{q}\right|_{E}$ attains its minimum at $p$. Since $E$ is extremal, it follows that $\measuredangle q p p^{\prime} \leq \frac{\pi}{2}$, a contradiction.

If $E$ is not compact, we use quasigeodesics according to Perelman-Petrunin [21]; see also [24, Section 5]. We may assume $E$ to be connected. Furthermore, by way of contradiction, let $p_{1}, p_{2} \in E$ such that $d_{F}\left(p_{1}\right)>d_{F}\left(p_{2}\right)$. According to the generalized Lieberman Lemma [24, 2.3.1], a shortest path $\gamma$ between $p_{1}$ and $p_{2}$ in the induced intrinsic metric of $E$ is a quasigeodesic of $M$. Hence, it can be extended inside $E$ for infinite time beyond $p_{2}$. Since $d_{F}$ is concave on quasigeodesics and strictly decreasing on $\gamma$ near $p_{2}$, we conclude that $E \cap F \neq \varnothing$, which contradicts the assumptions. Thus, $\left.d_{F}\right|_{E}$ is constant and we can proceed as in the first part of the proof. 
We now investigate superlevel sets of the distance function of a boundary stratum.

3.5 Lemma Let $M \in \operatorname{ALEX}^{n}(0)$ and let $F_{1}$ be a boundary stratum. Choose $s \in \mathbb{R}$ such that $M^{\prime}:=d_{F_{1}}^{-1}([s, \infty)) \neq \varnothing$. This implies that $M^{\prime} \in \operatorname{ALEX}^{m}(0)$, where $m \leq n$. Equality holds if and only if $s<\sup _{p \in M} d_{F_{1}}(p)$. In addition, if $F_{2}$ is another boundary stratum, we set $F_{2}^{\prime}:=F_{2} \cap M^{\prime}$ and obtain that $d_{F_{2}^{\prime}}=\left.d_{F_{2}}\right|_{M^{\prime}}$. Moreover, if $F_{2}^{\prime}$ is properly contained in $M^{\prime}$, it is a boundary stratum of $M^{\prime}$.

Proof The function $d_{F_{1}}$ is concave and therefore, $M^{\prime} \subseteq M$ is a convex subset. Since the dimension of $M$ is locally constant, collapse occurs exactly if $s=\sup d_{F_{1}}<\infty$. Let $p \in M^{\prime} \backslash F_{2}^{\prime}$ and $q \in F_{2}$ such that $d_{F_{2}}(p)=|p q|$. We claim that this implies $q \in F_{2}^{\prime}$ and hence $\left.d_{F_{2}}\right|_{M^{\prime}}=d_{F_{2}^{\prime}}$. First, we may assume that $q \notin F_{1}$, because $q \in F_{1}$ implies $p \in F_{1}$ and therefore the trivial case that $M^{\prime}=M$. Take $r \in F_{1}$ at minimal distance to $q \notin F_{1}$. By extremality of $F_{2}$ and the choice of $q$, we obtain that $\measuredangle p q r \leq \frac{\pi}{2}$ and hence $d_{q} d_{F_{1}}(\uparrow \stackrel{p}{q}) \leq 0$. Concavity of $d_{F_{1}}$ implies that $d_{F_{1}}(q) \geq d_{F_{1}}(p) \geq s$, which means that $q \in M^{\prime}$ and proves the claim.

Now it is clear that the following holds: If $p \in M^{\prime} \backslash F_{2}^{\prime}$ is given and the function $\left.d_{p}\right|_{F_{2}^{\prime}}$ attains its minimum at $q \in F_{2}^{\prime}$, we have that $\measuredangle p q x \leq \frac{\pi}{2}$ for all $x \in M^{\prime}$. Therefore, $F_{2}^{\prime}$ is an extremal set in $M^{\prime}$ (note that $F_{2}^{\prime}$ is closed). If $M^{\prime}$ is not collapsed, it is clear that $F_{2}^{\prime}$ has locally constant codimension 1 and hence is a boundary stratum. If $M^{\prime}$ is collapsed, but $F_{2}^{\prime} \subsetneq M^{\prime}$, let $t>0$ be small enough such that it is a regular value of the function $d_{F_{2}^{\prime}}$. By the Morse Lemma, the level set $Z:=d_{F_{2}^{\prime}}^{-1}(t)$ is of locally constant codimension 1 in $M^{\prime}$. We define a map $\psi: Z \rightarrow F_{2}$ like follows: For $z \in Z$ let $\psi(z)$ be the endpoint of some shortest path from $z$ to $F_{2}$. It is easy to see that $\psi$ is noncontracting, because a shortest path to a boundary stratum is perpendicular to it. The same holds in each superlevel set $d_{F_{2}}^{-1}\left(\left[t^{\prime}, \infty\right)\right)$ for $t^{\prime} \in(0, t)$ with boundary stratum $d_{F_{2}}^{-1}\left(t^{\prime}\right)$. Now, since $\psi(Z) \subseteq F_{2}^{\prime}$, we obtain that $\operatorname{dim} F_{2}^{\prime} \geq m-1$. However, $\operatorname{dim} F_{2}^{\prime}=m$ would imply $F_{2}^{\prime}=M^{\prime}$, since $F_{2}^{\prime}$ is extremal.

For the proof of the Splitting Theorem 1.2 both results of the lemma will be needed, the collapsed case and the non-collapsed one. In the latter case boundary strata are "shifted inwards" preserving their intersection behaviour like follows.

3.6 Lemma Let $M \in \operatorname{ALEX}^{n}(0)$ and let $F_{1}, \ldots, F_{\ell}$ be a stratification of $\partial M$. Let $s \in \mathbb{R}$ be given such that $M^{\prime}:=d_{F_{1}}^{-1}([s, \infty))$ has dimension $n$. We set $F_{1}^{\prime}:=d_{F_{1}}^{-1}(s)$ and $F_{i}^{\prime}:=F_{i} \cap M^{\prime}$ for $i=2, \ldots, \ell$. Then $F_{1}^{\prime}, \ldots, F_{\ell}^{\prime}$ is a stratification of $\partial M^{\prime}$ and the following holds:

$$
\bigcap_{i \in I} F_{i}=\varnothing \Longleftrightarrow \bigcap_{i \in I} F_{i}^{\prime}=\varnothing \quad \text { for each subset } I \subseteq\{1, \ldots, \ell\}
$$


Proof According to Lemma 3.5 the sets $F_{2}^{\prime}, \ldots F_{\ell}^{\prime}$ are boundary strata. We consider $F_{1}^{\prime}$ and again, for $s=0$ there is nothing to prove. Hence, we may assume that $s$ is a regular value of the function $d_{F_{1}}$. Indeed, local maxima of concave functions are in fact global, and $s=\sup d_{F_{1}}$ leads to collapse. Now the Morse Lemma implies that $F_{1}^{\prime}$ is the closure of points possessing neighborhoods in $M^{\prime}$ homeomorphic to $\mathbb{R}^{n-1} \times \mathbb{R}_{\geq 0}$. Therefore, $F_{1}^{\prime}$ is a boundary stratum of $M^{\prime}$. It is easy to see that the collection $F_{1}^{\prime}, \ldots, F_{\ell}^{\prime}$ is a stratification of $\partial M^{\prime}$. Finally, we investigate the intersections of boundary strata. The stratum $F_{1}$ plays a special role, so let $I \subseteq\{2, \ldots, \ell\}$ and $F:=\bigcap_{i \in I} F_{i}, F^{\prime}:=\bigcap_{i \in I} F_{i}^{\prime}$.

If $F=\varnothing$, clearly also $F^{\prime}=\varnothing$. Thus, let $F \neq \varnothing$ and consider the following two cases. If $F_{1} \cap F=\varnothing$, we can apply Lemma 3.4, because $F$ is an extremal set. We obtain that $d_{F_{1}}(F)=\max d_{F_{1}}>s$ and therefore $F^{\prime}=F$. If $F_{1} \cap F \neq \varnothing$, let $p \in F_{1} \cap F$ and consider the gradient curve $\alpha_{p}$ of the function $d_{F_{1}}$. Since $\alpha_{p}(t) \in F$ for all $t \geq 0$, there exists $T>0$ such that $d_{F_{1}}\left(\alpha_{p}(T)\right)=s$. This implies that $\alpha_{p}(T) \in F_{1}^{\prime} \cap F^{\prime} \neq \varnothing$ and completes the proof.

3.7 Corollary For $M \in \operatorname{ALEX}^{n}(0)$ let $F_{1}, \ldots, F_{\ell}$ be a stratification of $\partial M$ and $s_{1}, \ldots, s_{\ell} \in \mathbb{R}$ such that $M^{\prime}:=d_{F_{1}}^{-1}\left(\left[s_{1}, \infty\right)\right) \cap \ldots \cap d_{F_{\ell}}^{-1}\left(\left[s_{\ell}, \infty\right)\right)$ is non-collapsed. Then $\partial M^{\prime}$ possesses the stratification $\partial M^{\prime}=F_{1}^{\prime} \cup \ldots \cup F_{\ell}^{\prime}$ with boundary strata $F_{i}^{\prime}:=d_{F_{i}}^{-1}\left(s_{i}\right) \cap M^{\prime}$. Moreover, the intersection of any collection of the strata $F_{i}$ is empty if and only if the intersection of the corresponding strata $F_{i}^{\prime}$ is empty.

Proof This follows by iterated use of Lemma 3.6 and the fact that the order of taking superlevel sets is irrelevant according to Lemma 3.5.

\section{Intersections of boundary strata}

In order to investigate boundary strata, it is often useful not to work in the space $M$ itself but in slightly smaller superlevel sets. Thereafter, we pass to the Hausdorff limits, which behave like expected.

4.1 Lemma Let $M \in \operatorname{ALEX}^{n}(0)$ and let $E, F_{1}, \ldots, F_{k}$ be a stratification of $\partial M$. We choose a sequence $\left(s_{i}\right)$ decreasing to 0 such that all $M_{i}:=d_{E}^{-1}\left(\left[s_{i}, \infty\right)\right)$ are not collapsed. We set $E_{i}:=d_{E}^{-1}\left(s_{i}\right)$ and let $F=\bigcap_{\ell \in I} F_{\ell}$ be the intersection of boundary strata for some index set $I \subseteq\{1, \ldots, k\}$. Then we have the Hausdorff convergences $M_{i} \stackrel{i \rightarrow \infty}{\longrightarrow} M, E_{i} \stackrel{i \rightarrow \infty}{\longrightarrow} E$ and $E_{i} \cap F \stackrel{i \rightarrow \infty}{\longrightarrow} E \cap F$. 
Proof Since $s_{i}$ is decreasing, we have that $M_{i} \subseteq M_{i+1}$ for all $i$. Thus, the convergence $M_{i} \rightarrow M$ is clear. Now, if $\varepsilon>0$ is given, choose $i$ big enough such that $\max \left\{s_{i}, d_{H}\left(M_{i}, M\right)\right\}<\varepsilon$, where $d_{H}$ denotes the Hausdorff distance. Then we have by definition $\left|p M_{i}\right|<\varepsilon$ for all $p \in M$, which implies $\left|p E_{i}\right|<\varepsilon$ for all $p \in E$. On the other hand we have $|q E|=s_{i}<\varepsilon$ for all $q \in E_{i}$ and hence, $E_{i} \rightarrow E$. For the remaining convergence we show that $E \cap F$ is exactly the set of limit points of sequences $\left(p_{i}\right)$ with $p_{i} \in E_{i} \cap F$. Indeed, if $\left(p_{i}\right)$ is such a sequence converging to $p \in M$, then clearly $p \in E \cap F$, since $E_{i} \rightarrow E$ and $F$ is closed. Vice versa, let $q \in E \cap F$ and let $\alpha_{q}$ be the gradient curve of the function $d_{E}$ starting at $q$. Hence, the gradient curve $\alpha_{q}$ stays in $F$. By setting $q_{i}:=\alpha_{q} \cap E_{i}$ we obtain a sequence $q_{i} \rightarrow q$ with $q_{i} \in E_{i} \cap F$. Now it is clear that $E \cap F$ is the Hausdorff limit of $E_{i} \cap F$, since this holds by Blaschke's Compactness Theorem (see for example Burago-Burago-Ivanov [2, Theorem 7.3.8]) inside any compact ball.

We are now able to prove the first part of Theorem 1.4 using the Morse Lemma and the Stability Theorem as well as their relative versions.

4.2 Proposition Let $M \in \operatorname{ALEX}^{n}(0)$ and let $F_{1}, \ldots, F_{k}$ be boundary strata. If the intersection $F_{1} \cap \ldots \cap F_{k}$ is nonempty, it is an extremal set of locally constant dimension $n-k$ and $k \leq n$ holds necessarily.

Proof We use induction over $k$. The base step $k=1$ is clear, since a boundary stratum $F \subseteq M$ induces for $p \in F$ the boundary stratum $\Sigma_{p} F \subseteq \Sigma_{p}$. Thus, induction over dimension shows that an extremal set is a boundary stratum if, and only if, it has locally constant codimension 1 .

Now assume that $F_{1} \cap \ldots \cap F_{k} \neq \varnothing$ and that, by induction assumption, $F_{1} \cap \ldots \cap F_{k}$ has locally constant dimension $n-k+1$. First of all, for any $p \in F_{1} \cap \ldots \cap F_{k-1}$ the gradient curve of the function $d_{F_{k}}$ immediately leaves the set $F_{k}$, but stays in $F_{1} \cap \ldots \cap F_{k-1}$. This implies that $\operatorname{dim}\left(F_{1} \cap \ldots \cap F_{k-1}\right) \geq 1$ and therefore $k \leq n$. Let $s>0$ be small enough such that $d_{F_{k}}^{-1}([s, \infty))$ is not collapsed. In other words, $s$ is a regular value for $d_{F_{k}}$. By the relative Morse Lemma, the set $F_{1} \cap \ldots \cap F_{k-1} \cap d_{F_{k}}^{-1}(s)$ has dimension $n-k$, possibly not locally constant. We know, however, that $F_{1} \cap \ldots \cap F_{k-1}$ has locally constant dimension, which implies that the relative Morse Lemma works like the original Morse Lemma. Namely, $F_{1} \cap \ldots \cap F_{k-1} \cap d_{F_{k}}^{-1}(s)$ is an MCS-space instead of only an $\widehat{\text { MCS }}$-space, compare Perelman-Petrunin [20, Section 2]. This implies that the space has locally constant dimension.

Taking Lemma 3.6 into account, the induction step is proved for the superlevel set $d_{F_{k}}^{-1}([s, \infty))$. Now we choose the values for $s$ as a sequence descending to 0 . By 
Lemma 4.1, boundary strata and their intersections in the superlevel sets converge in the Hausdorff sense to the corresponding intersections in $M$. If $M$ is compact, the relative Stability Theorem by Kapovitch [7, Theorem 9.2] implies that the converging extremal sets in question are homeomorphic to their limit. Thus, also the latter one has locally constant dimension $n-k$. In the noncompact case, the (relative) Stability Theorem holds for pointed Gromov-Hausdorff limits, compare [7, Theorem 7.11]. This implies in our setting that the converging extremal sets are locally homeomorphic to their limit. Hence, also in this case the induction step is proved.

4.3 Corollary For $\Sigma \in \operatorname{ALEX}^{n}(1)$ let $F_{1}, \ldots, F_{k}$ be boundary strata. Then we have that $F_{1} \cap \ldots \cap F_{k} \neq \varnothing$ if and only if $k \leq n$.

Proof This follows immediately from Proposition 4.2 and Petrunin's version of Frankel's Theorem [23, Theorem 3.2].

Note that Proposition 4.2 can also be proved using the spaces of directions, which requires double induction over $n$ and $k$, see [31, Theorem 2.18]. A similar method like above, however, will be used to prove the following fact.

4.4 Proposition Let $M \in \operatorname{ALEX}^{n}(0)$ and let $F_{1}, \ldots, F_{k}$ be boundary strata. Assume that $F:=F_{1} \cap \ldots \cap F_{k} \neq \varnothing$ and let $p \in F$ such that $T_{p} F \approx \mathbb{R}^{n-k-1} \times \mathbb{R}_{\geq 0}$. Then the point $p$ is contained in some boundary stratum distinct from $F_{1}, \ldots, F_{k}$, in other words $p \in \overline{\partial M \backslash\left(F_{1} \cup \ldots \cup F_{k}\right)}$. In particular, if there is no additional boundary stratum intersecting with $F_{1} \cap \ldots \cap F_{k}$, such points $p$ do not exist.

Proof The assertion is proved via induction on $k$. As base step we consider $k=0$ and define $F$ to equal $M$. Then Perelman's characterization of the boundary implies that $p \in \partial M$.

For the induction step, let $p \in F$ with $T_{p} F \approx \mathbb{R}^{n-k-1} \times \mathbb{R}_{\geq 0}$ and assume, by way of contradiction, that $p$ lies in no other boundary stratum. Hence, we can assume that $\partial M=F_{1} \cup \ldots \cup F_{k}$. Indeed, otherwise consider the doubling $\bar{M}$ obtained by gluing along the boundary stratum $\overline{\partial M \backslash\left(F_{1} \cup \ldots \cup F_{k}\right)}$. This does not affect $T_{p} F=T_{p} \bar{F}$ by the assumption on $p$. Now choose $s>0$ small enough such that it is a regular value for $d_{F_{k}}$. We consider the set $F^{\prime}:=F_{1} \cap \ldots \cap F_{k-1} \cap d_{F_{k}}^{-1}(s)$ and claim that there is no open subset $U \stackrel{\circ}{\subseteq} F^{\prime}$ homeomorphic to $\mathbb{R}^{n-k-1} \times \mathbb{R}_{\geq 0}$. Assume the contrary and let $q \in U$. The function $d_{F_{k}}$ is admissible and regular near $q$ and the set $F_{1} \cap \ldots \cap F_{k-1}$ is extremal. Hence, the relative local fibration theorem gives that $q$ has a neighborhood $V \stackrel{\circ}{\subseteq} F_{1} \cap \ldots \cap F_{k-1}$ satisfying $V \approx U \times \mathbb{R} \approx \mathbb{R}^{n-k} \times \mathbb{R}_{\geq 0}$. By 
the induction assumption it follows that $q$ lies in some boundary stratum distinct from $F_{1}, \ldots, F_{k-1}$. This implies $q \in F_{k}$, a contradiction to the definition of $F^{\prime}$.

The claim still holds if we choose the values of $s$ descending to 0 . By Lemma 4.1 and the relative Stability Theorem, we have that $F \approx F^{\prime}$ for $s$ small enough and if $M$ is compact. In the noncompact case we may consider everything inside an appropriate compact ball. By the choice of $p$, there is some open subset in $F$ homeomorphic to $\mathbb{R}^{n-k-1} \times \mathbb{R}_{\geq 0}$. Hence, such subset exists also in $F^{\prime}$, a contradiction to the claim. Thus, the induction step is proved.

As another structure result, in positive curvature intersections of boundary strata turn out to be connected (unless they are 0-dimensional).

4.5 Lemma Let $\Sigma \in \operatorname{ALEX}^{n}(1)$ and let $F_{1}, \ldots, F_{k}$ be boundary strata with $k<n$, $n \geq 2$. Then the intersection $F_{1} \cap \ldots \cap F_{k}$ is connected.

Proof First, recall that $F_{1} \cap \ldots \cap F_{k} \neq \varnothing$ by Corollary 4.3. Now we use induction on $k$. The base step $k=1$ is clear by Frankel's/Petrunin's Theorem. Indeed, $\operatorname{dim} F_{1}=n-1$ and $2(n-1) \geq n$ since $n \geq 2$. Therefore, two connected components of $F_{1}$ would intersect.

For the proof of the induction step let $p, q \in F_{1} \cap \ldots \cap F_{k}$. Since $d_{F_{1}}$ is strictly concave, the subset in $\Sigma$ where $d_{F_{1}}$ attains its maximum consists of one point only. If $z_{1}$ denotes this point, we have that $z_{1} \in F_{2} \cap \ldots \cap F_{k}$, because all gradient curves of the function $d_{F_{1}}$ end at the point $z_{1}$. In particular, there is a curve $\gamma \subseteq F_{2} \cap \ldots \cap F_{k}$ from $p$ to $q$. We claim that $\gamma$ can be chosen such that $z_{1} \notin \gamma$. Assume the contrary, then for each neighborhood $U \subseteq F_{2} \cap \ldots \cap F_{k}$ of $z_{1}$ the set $U \backslash\left\{z_{1}\right\}$ is not pathconnected. This implies that $K\left(\Sigma_{z_{1}} F_{2} \cap \ldots \cap \Sigma_{z_{1}} F_{k}\right) \backslash$ apex is not path-connected and therefore also $\Sigma_{z_{1}} F_{2} \cap \ldots \cap \Sigma_{z_{1}} F_{k}$ is not path-connected. By induction assumption, $\Sigma_{z_{1}} F_{2} \cap \ldots \cap \Sigma_{z_{1}} F_{k}$ is connected and hence also path-connected, since it is a stratified manifold. This contradiction proves the claim.

Let $x \in \Sigma \backslash\left(F_{1} \cup\left\{z_{1}\right\}\right)$. The concavity of $d_{F_{1}}$ implies that $\left|\Uparrow_{x}^{F_{1}} \Uparrow_{x}^{z_{1}}\right|>\frac{\pi}{2}$. Therefore, the gradient $\nabla_{x} f$ of the function $f:=d_{z_{1}}^{2}$ is non-zero. Thus, the gradient flow of $f$ pushes $\gamma$ into the extremal subset $F_{1} \cap \ldots \cap F_{k}$. We assume that there is some $T \in(0, \infty)$ such that $\delta:=\Phi_{f}^{T}(\gamma) \subseteq F_{1} \cap \ldots \cap F_{k}$. If $\alpha_{p}, \alpha_{q}$ denote the gradient curves of $f$ starting at $p$ and $q$, respectively, the curve $\delta$ connects the points $\alpha_{p}(T)$ and $\alpha_{q}(T)$. Hence $F_{1} \cap \ldots \cap F_{k}$ is connected. If such finite $T$ does not exist, perform the construction from above in the space $\Sigma^{\prime}=d_{F_{1}}^{-1}([s, \infty))$ for $s>0$ small enough. It is clear that in the space $\Sigma^{\prime}$ an appropriate $T$ exists. Now let $s \searrow 0$ and apply Lemma 4.1, which gives that $F_{1} \cap \ldots \cap F_{k}$ is connected as Hausdorff limit of the corresponding connected sets $F_{1}^{\prime} \cap \ldots \cap F_{k}^{\prime}$. 


\section{Maximum sets}

We already know that the set where the distance function of a boundary stratum attains its maximal value is a convex set. It inherits boundary strata from the ambient space if it is not contained in the strata in question. In this section we investigate such maximum sets more closely.

5.1 Lemma Let $M \in \operatorname{ALEX}^{n}(0)$ and let $F_{1}, \ldots, F_{k+1}$ be boundary strata such that the following holds.

(1) $F_{1} \cap \ldots \cap F_{k+1}=\varnothing$,

(2) $F_{1} \cap \ldots \cap \widehat{F}_{i} \cap \ldots \cap F_{k+1} \neq \varnothing \quad \forall i \in\{1, \ldots, k+1\}$.

For each $i \in\{1, \ldots, k+1\}$ the maximum $a_{i}:=\max _{p \in M} d_{F_{i}}(p)$ exists and we set $A_{i}:=\left\{p \in M \mid d_{F_{i}}(p)=a_{i}\right\}$. Let $p \in A_{i}$ and $q \in M$ such that for all $j \neq i$ we have that $d_{F_{j}}(q)<d_{F_{j}}(p)$ if $d_{F_{j}}(p)>0$ and $d_{F_{j}}(q)=0$ if $d_{F_{j}}(p)=0$. Then also $q \in A_{i}$. Moreover, it follows that $A_{i} \subseteq F_{1} \cup \ldots \cup \widehat{F}_{i} \cup \ldots \cup F_{k+1}$.

Proof We show the assertion for $A_{k+1}$. First of all, the assumptions and Lemma 3.4 imply that $d_{F_{k+1}}$ attains its maximum on $F_{1} \cap \ldots \cap F_{k}$, that is $F_{1} \cap \ldots \cap F_{k} \subseteq A_{k+1}$. According to Corollary 3.7, the analog is true for each superlevel set as long as no collapse occurs. For $j=1, \ldots, k$ we set $s_{j}:=d_{F_{j}}(p)$ and $t_{j}:=d_{F_{j}}(q)$. Now we consider the space $M^{\prime}:=d_{F_{1}}^{-1}\left(\left[t_{1}, a_{1}\right]\right) \cap \ldots \cap d_{F_{k}}^{-1}\left(\left[t_{k}, a_{k}\right]\right)$, which is not collapsed since $t_{j}<s_{j}$ or $t_{j}=s_{j}=0$. We have that $q \in F_{1}^{\prime} \cap \ldots \cap F_{k}^{\prime}$ and again by Lemma 3.4, the function $\left.d_{F_{k+1}}\right|_{M^{\prime}}$ attains its maximum at $q$. On the other hand we, have that $p \in M^{\prime}$ and therefore $a_{k+1}=d_{F_{k+1}}(p) \leq d_{F_{k+1}}(q) \leq a_{k+1}$. This implies everywhere equality and hence $q \in A_{k+1}$.

Assume now, by way of contradiction, that $s_{j}>0$ for all $j=1, \ldots, k$. Let $z \in$ $F_{1} \cap \ldots \cap F_{k}$ and $r>0$ satisfying $r<s_{j}$ for all $j \in\{1, \ldots, k\}$. Then, by the first part, the ball $B_{r}(z)$ (taken in $M$ ) is contained in $A_{k+1}$, a contradiction to the definition of $A_{k+1}$. Hence, for each $p \in A_{k+1}$ there exists some index $i$ such that $d_{F_{i}}(p)=0$. In other words, $A_{k+1} \subseteq F_{1} \cup \ldots \cup F_{k}$.

The last statement enables the proof of the following result, which in turn provides the remaining part of Theorem 1.4.

5.2 Lemma Let $M \in \operatorname{ALEX}^{n}(0)$ and let $F_{1}, \ldots, F_{k+1}$ be boundary strata. Then the following constellation is impossible: All intersections of $k-1$ boundary strata $F_{i}$ are nonempty, while all intersections of $k$ boundary strata are empty. 
Proof Assume, by way of contradiction, there is such constellation. Note that only for $k \geq 2$ there is something to prove. Let $A_{k+1}$ as in Lemma 5.2 and set $G_{i}:=$ $F_{1} \cap \ldots \cap \hat{F}_{i} \cap \ldots \cap F_{k}$ for $i=1, \ldots, k$. By assumption there are points $p_{i} \in G_{i}$ for each $i \in\{1, \ldots, k\}$ and these points are pairwise distinct. In addition, we have that $G_{i} \cap F_{k+1}=\varnothing$ and therefore, by Lemma 3.4, that $G_{i} \subseteq A_{k+1}$ for $i=1, \ldots, k$. Moreover, by applying Lemma 5.1 to the subcollection $F_{2}, \ldots, F_{k+1}$, we obtain that $A_{k+1} \subseteq F_{2} \cup \ldots \cup F_{k}$. Let $q_{1} \in p_{1} p_{2}$ be some interior point. Since $A_{k+1}$ is convex, we have that $q_{1} \in A_{k+1}$. The fact that $p_{1} \notin F_{1}$ and $p_{2} \notin F_{2}$ implies that $q_{1} \in\left(F_{2} \cup \ldots \cup F_{k}\right) \backslash\left(F_{1} \cup F_{2}\right)$, because all $F_{i}$ are extremal. Now let $q_{2} \in q_{1} p_{3}$ be some interior point and obtain that $q_{2} \in\left(F_{2} \cup \ldots \cup F_{k}\right) \backslash\left(F_{1} \cup F_{2} \cup F_{3}\right)$ and iterate. This process leads to some point $q_{k-1} \in q_{k-2} p_{k}$ satisfying the condition $q_{k-1} \in\left(F_{2} \cup \ldots \cup F_{k}\right) \backslash\left(F_{1} \cup \ldots \cup F_{k}\right)$, a contradiction.

Maximum sets lying in some boundary strata may have additional boundary strata not coming from the ambient space. Namely, there may be topological boundary as well. In the following we make this precise.

5.3 Definition Let $(X, d)$ be a metric space and $A \subseteq B \subseteq X$. The topological boundary $\mathrm{Bd}_{B} A$ of $A$ in $B$ is defined as

$$
\operatorname{Bd}_{B} A:=\left\{x \in B \mid B_{r}(x) \cap A \neq \varnothing \text { and } B_{r}(x) \cap(B \backslash A) \neq \varnothing \quad \forall r>0\right\},
$$

where the balls are taken in $X$.

5.4 Lemma Let $M \in \operatorname{ALEX}^{n}(0)$ and let $F_{1}, \ldots, F_{k}$ be boundary strata with $k<n$. Let $A \subseteq M$ be a convex subset satisfying $A \subseteq F_{1} \cap \ldots \cap F_{k}$ and $\operatorname{dim} A=n-k$. Then $p \in \operatorname{Bd}_{F_{1} \cap \ldots \cap F_{k}} A$ implies that $p \in \partial A$.

Proof We use induction on $m:=n-k$. The base step $m=1$ is trivial by definition of $\partial A$. For $m \geq 2$ let $p \in \operatorname{Bd}_{F_{1} \cap \ldots \cap F_{k}} A$ and let $\varepsilon>0$. We endow the extremal set $F_{1} \cap \ldots \cap F_{k}$ with the induced intrinsic metric denoted by $\hat{d}$. Recall that $d$ and $\hat{d}$ are locally bi-Lipschitz equivalent according to Perelman-Petrunin [20, Corollaries 3.2]. We choose $q \in\left(F_{1} \cap \ldots \cap F_{k}\right) \backslash A$ such that $\hat{d}(p, q) \leq \frac{\varepsilon}{2}$. Let $x \in A$ be a point with minimal distance $\widehat{d}(x, q)$. Let $\gamma$ be a shortest path in $F_{1} \cap \ldots \cap F_{k}$ from $x$ to $q$. The choice of $x$ implies that $\gamma^{+}(0) \notin \Sigma_{x} A$. In other words, the convex subset $\Sigma_{x} A \subseteq \Sigma_{x}$ satisfies $\Sigma_{x} A \subsetneq \Sigma_{x}\left(F_{1} \cap \ldots \cap F_{k}\right)$. By Lemma 4.5 , the set $\Sigma_{x}\left(F_{1} \cap \ldots \cap F_{k}\right)=$

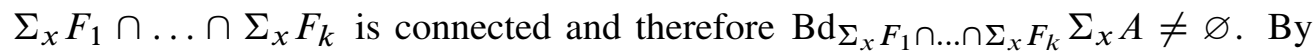
the induction assumption we conclude that $\partial \Sigma_{x} A \neq \varnothing$ and hence $x \in \partial A$. Since $d(x, p) \leq \widehat{d}(x, p) \leq \varepsilon$ and $\partial A$ is closed, this implies that $p \in \partial A$ as desired. 
5.5 Remark The statement remains true for $k=0$, that is, if $A \subseteq M$ is a convex subset of full dimension. A proof will be carried out in the upcoming book on Alexandrov geometry by Alexander, Kapovitch and Petrunin. The proof of the lemma above is an adapted version of their one.

Under the assumptions of our Splitting Theorem 1.2 we observe the interesting fact that the maximum sets $A_{i}=\left\{p \mid d_{F_{i}}(p)=\max d_{F_{i}}\right\}$ essentially look the same as the space $M$ itself. This means, if $\partial A_{i}$ is not empty, it contains $m+1<k+1$ boundary strata fulfilling the same intersection conditions as the strata of $\partial M$. This property is crucial for the proof of our Splitting Theorem, since we may assume by an induction argument that inside $A_{i}$ everything is already proved.

5.6 Proposition Let $M \in \operatorname{ALEX}^{n}(0)$ and let $F_{1}, \ldots, F_{k+1}$ be a stratification of $\partial M$ such that $F_{1} \cap \ldots \cap F_{k+1}=\varnothing$, while $F_{1} \cap \ldots \cap \widehat{F}_{\ell} \cap \ldots \cap F_{k+1} \neq \varnothing$ for $\ell=1, \ldots, k+1$. We set $a_{\ell}:=\max d_{F_{\ell}}$ and $A_{\ell}:=\left\{p \in M \mid d_{F_{\ell}}(p)=a_{\ell}\right\}$. Then $\partial A_{\ell} \neq \varnothing$ holds if and only if $A_{\ell} \supsetneq F_{1} \cap \ldots \cap \widehat{F}_{\ell} \cap \ldots \cap F_{k+1}$. In this case let $I \subseteq\{1, \ldots, k+1\}$ be the maximal set of indices such that $A_{\ell} \subseteq \bigcap_{i \in I} F_{i}=: F$. Then $1 \leq|I|<k$ and a stratification of $\partial A_{\ell}$ is given by the strata $A_{\ell} \cap F_{i}$ for $i \in\{1, \ldots, k+1\} \backslash(I \cup\{\ell\})$ plus the stratum $\mathrm{Bd}_{F} A_{\ell}$. These strata have an analog intersection behaviour as the $F_{i}$, namely all together have empty intersection, while all minus one do intersect. In addition, the dimension of $A_{\ell}$ satisfies $\operatorname{dim} A_{\ell}=\operatorname{dim} F=n-|I|$.

Proof The assertion will be proved for $A_{k+1}$. By Lemma 3.4, we have that $F_{1} \cap$ $\ldots \cap F_{k} \subseteq A_{k+1}$. If equality holds, Proposition 4.4 implies that $\partial A_{k+1}=\varnothing$. Hence, we assume that $F_{1} \cap \ldots \cap F_{k} \subsetneq A_{k+1}$. Let $p \in A_{k+1}$ be a point such that $d_{F_{i}}(p) \neq 0$ for a maximal number of indices $i \in\{1, \ldots, k\}$. By renumbering we may assume that $d_{F_{i}}(p) \neq 0$ for $i=1, \ldots, m$ and $d_{F_{i}}(p)=0$ for $i=m+1, \ldots, k$. According to Lemma 5.1, we have that $m<k$. We claim that $A_{k+1} \subseteq F_{m+1} \cap \ldots \cap F_{k}$. Assume, by way of contradiction, there exists $q \in A_{k+1}$ and $j \in\{m+1, \ldots, k\}$ with $d_{F_{j}}(q) \neq 0$. Recall that $F_{j}$ is an extremal set. Thus, any inner point $r$ of some shortest path $p q$ fulfills $d_{F_{i}}(r) \neq 0$ for $i \in\{1, \ldots, m\} \cup\{j\}$. But $r$ also lies in the convex set $A_{k+1}$, contradicting the choice of $p$. This means that $I=\{1, \ldots, m\}$. By the claim and Proposition 4.2, we obtain that $\operatorname{dim} A_{k+1} \leq \operatorname{dim}\left(F_{m+1} \cap \ldots \cap F_{k}\right)=n-(k-m)$. The converse inequality follows by Lemma 5.1. Indeed, if we set $s_{i}:=d_{F_{i}}(p)$ for $i=1, \ldots m$, we have that $d_{F_{1}}^{-1}\left(\left[0, s_{1}\right)\right) \cap \ldots \cap d_{F_{m}}^{-1}\left(\left[0, s_{m}\right)\right) \cap F_{m+1} \cap \ldots \cap F_{k} \subseteq A_{k+1}$. Now we set $G_{i}:=A_{k+1} \cap F_{i}$ for $i=1, \ldots, m$. According to Lemma 3.5 (applied for $s=a_{k+1}$, hence $M^{\prime}=A_{k+1}$ ), the sets $G_{i}$ are boundary strata of $A_{k+1}$. We have to show that there exists the additional boundary stratum $G_{m+1}:=\operatorname{Bd}_{F_{m+1}} \cap \ldots \cap F_{k} A_{k+1}$ and that the strata intersect like stated in the Proposition. Let $p \in \partial A_{k+1}$. Assume 
that $p \notin G_{m+1}$, which implies that $T_{p} A_{k+1}=T_{p}\left(F_{m+1} \cap \ldots \cap F_{k}\right)$. According to Proposition 4.4, we have that $p \in G_{i}$ for some $i \in\{1, \ldots, m\}$. Indeed, this is true if $T_{p} A_{k+1} \approx \mathbb{R}^{n-(k-m)-1} \times \mathbb{R}_{+}$and such points are dense in $\partial A_{k+1} \backslash G_{m+1}$. Therefore it follows that $\partial A_{k+1} \subseteq G_{1} \cup \ldots \cup G_{m+1}$. It is clear that $G_{m+1} \neq \varnothing$, because each limit point of some gradient curve to the function $d_{F_{k+1}}$ starting (and hence staying) in $F_{m+1} \cap \ldots \cap F_{k}$ lies in $G_{m+1}$. In order to refine this argument, the same holds if the gradient curve starts in $F_{1} \cap \ldots \cap \widehat{F}_{j} \cap \ldots \cap F_{k}$, for each $j \in\{1, \ldots, m\}$. By slightly "shifting" the strata $F_{1}, \ldots, F_{m}$, that is, performing that construction in superlevel sets $M^{\prime}$ close to $M$, we conclude that there are points in $G_{m+1}$ arbitrary close to $G_{1} \cap \ldots \cap \widehat{G}_{j} \cap \ldots \cap G_{m}$, but not contained in any $G_{1}, \ldots, G_{m}$, for each $j \in\{1, \ldots, m\}$. According to Lemma 5.4, we have that $G_{m+1} \subseteq \partial A_{k+1}$. Hence, it follows that $\overline{\partial A_{k+1} \backslash\left(G_{1} \cup \ldots \cup G_{m}\right)}$ is nonempty and therefore a boundary stratum. Moreover, we have that $\overline{\partial A_{k+1} \backslash\left(G_{1} \cup \ldots \cup G_{m}\right)} \subseteq G_{m+1}$. In order to see equality, consider the doubling $\bar{M}$ by gluing along $F_{1} \cup \ldots \cup F_{m}$. The set $\bar{A}_{k+1}$ is a convex set of $\bar{M}$. Its boundary $\partial \bar{A}_{k+1}$ coincides on the one hand with the topological boundary $\operatorname{Bd}_{\bar{F}_{m+1} \cap \ldots \cap \bar{F}_{k}} \bar{A}_{k+1}=\bar{G}_{m+1}$ and on the other hand with the doubling of the stratum $\overline{\partial A_{k+1} \backslash\left(G_{1} \cup \ldots \cup G_{m}\right)}$. Similar doubling procedures show that $G_{1} \cup \ldots \cup G_{m+1}$ is indeed a stratification of $\partial A_{k+1}$, that is, there is no intersection $G_{i} \cap G_{j}, i \neq j$ which is itself a boundary stratum.

As proved above, we have that $G_{1} \cap \ldots \cap \widehat{G}_{j} \cap \ldots \cap G_{m} \cap G_{m+1} \neq \varnothing$ for all $j=1, \ldots, m$. Moreover, by $G_{1} \cap \ldots \cap G_{m}=F_{1} \cap \ldots \cap F_{m} \cap A_{k+1} \subseteq F_{1} \cap \ldots \cap F_{k} \subseteq$ $F_{1} \cap \ldots \cap F_{m} \cap A_{k+1}$ we conclude that $G_{1} \cap \ldots \cap G_{m}=F_{1} \cap \ldots \cap F_{k} \neq \varnothing$. It remains to show that $G_{1} \cap \ldots \cap G_{m+1}=\varnothing$. Again, choose $p \in A_{k+1}$ such that $s_{i}:=d_{F_{i}}(p)>0$ for all $i=1, \ldots, m$. Then $d_{F_{1}}^{-1}\left(\left[0, s_{1}\right)\right) \cap \ldots \cap d_{F_{m}}^{-1}\left(\left[0, s_{m}\right)\right) \cap F_{m+1} \cap \ldots \cap F_{k} \subseteq A_{k+1}$ is a neighborhood of the set $G_{1} \cap \ldots \cap G_{m}$. In particular, this neighborhood does not contain any points of $G_{m+1}$.

Note that the Proposition requires that $F_{1}, \ldots, F_{k+1}$ is indeed a stratification of $\partial M$. However, as mentioned before, if there are additional boundary strata, we may glue them away. In this form we will approach the proof of our Splitting Theorem.

\section{Assumptions for the subsequent sections}

From now on until otherwise stated we use the following assumptions and notation.

6.1 Notation The space $M$ is given as in the Splitting Theorem 1.2 with $k=\ell$. Hence, $F_{1}, \ldots, F_{k+1}$ is a stratification of $\partial M$ such that any $k$ strata intersect, but not all $k+1$. Moreover, we set $f_{i}:=d_{F_{i}}, a_{i}:=\max _{p \in M} f_{i}(p)$ and $A_{i}:=\left\{p \in M \mid f_{i}(p)=a_{i}\right\}$. 
By an induction argument we assume that the Splitting Theorem holds for all spaces fulfilling the above assumptions for a boundary stratification with less than $k+1$ strata. The base step has to be proved directly.

6.2 Proposition If $k=1$, then $F_{1}$ and $F_{2}$ are isometric convex subsets of dimension $n$, and $M$ is isometric to the product $F_{1} \times[0, d]$ where $d=\left|F_{1} F_{2}\right|$.

This fact is not difficult to prove and has been known to be true by a large number of experts in the field. Probably, it was not written up anywhere except in the author's $\mathrm{PhD}$ thesis [31, Theorem 3.3]. For a detailed proof we refer there and only sketch it briefly here.

Sketch of proof Lemma 3.4 and Lemma 5.1 imply that $F_{1}=A_{2}$ and $F_{2}=A_{1}$ are convex and equidistant sets. According to Proposition 4.4, they have no boundary. We set $d:=a_{1}=a_{2}$. The arguments can be repeated for any non-collapsed superlevel set $f_{i}^{-1}([s, d])$. Hence, $M$ is fibrated into souls of dimension $n-1$. It is easy to see that a dual fibration is given by shortest paths from any $p \in F_{1}$ to $F_{2}$ (or vice versa; these paths are unique). The dual fibers are also equidistant and intersect perpendicularly with the souls. Hence, the space $M$ carries the product metric where the factors are given by an arbitrary soul and an arbitrary dual fiber.

\section{Fibration into isometric souls}

7.1 Proposition The space $M$ is fibrated into totally convex subsets of dimension $n-k$ without boundary. On each such set all functions $f_{i}$ are constant, $i=1, \ldots, k+1$. All intersections $F_{1} \cap \ldots \cap \widehat{F}_{i} \cap \ldots \cap F_{k+1}$ belong to the fibration. Moreover, each set $A_{i}$ possesses a fibration which coincides with the one of $M$ on $A_{i}$.

7.2 Definition The elements of the fibration in Proposition 7.1 are called souls of $M$. We denote by $S(p)$ the soul containing the point $p \in M$.

7.3 Remark The term "soul" for a totally convex subset $S \subseteq M$ without boundary suggests (according to the Soul Theorem by Cheeger and Gromoll) that $M$ can be retracted onto $S$. This will be proved later. Note also that Proposition 7.1 implies that the fibration is compatible with all (intersections of) superlevel sets. That is, if $M^{\prime}:=f_{1}^{-1}\left(\left[s_{1}, a_{1}\right]\right) \cap \ldots \cap f_{k+1}^{-1}\left(\left[s_{k+1}, a_{k+1}\right]\right)$ is non-collapsed, its fibration coincides with the fibration of $M$ on $M^{\prime}$. The statement about the sets $A_{i}$ carries over to respective sets $A_{i}^{\prime} \subseteq M^{\prime}$. 
Proof of Proposition 7.1 We fix some $i \in\{1, \ldots, k+1\}$. If $F_{1} \cap \ldots \cap \widehat{F}_{i} \cap \ldots \cap$ $F_{k+1}$ coincides with $A_{i}$, it is a totally convex set. Its dimension equals $n-k$ by Proposition 4.2. Otherwise, according to Proposition 5.6, the boundary $\partial A_{i}$ consists of $m+1$ strata for some $1 \leq m<k$. In addition, some intersection of $m$ strata of $\partial A_{i}$ coincides with $F_{1} \cap \ldots \cap \widehat{F}_{i} \cap \ldots \cap F_{k+1}$. Consequently, this set is totally convex, since by induction assumption the statement is proved for $A_{i}$.

In order to obtain a soul $S(p)$ through an arbitrary point $p \in M$, we do the following. We set $s_{\ell}:=f_{\ell}(p)$ for $\ell=1, \ldots, k+1$. If there is an index $j$ such that $s_{j}=a_{j}$, then $p \in A_{j}$ and the statement follows like above. If such an index does not exist, $p$ is in particular not contained in any intersection of $k$ boundary strata. Hence, there is an index (in fact, at least two indices) $j$ such that $0<s_{j}<a_{j}$. Then we consider the superlevel set $M^{\prime}:=f_{j}^{-1}\left(\left[s_{j}, a_{j}\right]\right)$ and iterate the procedure, which clearly terminates. However, it may happen that $p$ lies in several maximum sets $A_{j}$. We have to clarify that $S(p)$ does not depend on the choice of $j$, because such dependencies could complicate further proofs, especially in view of the inductive arguments.

Assume that, say, $p \in A_{1} \cap A_{2}$. According to Lemma 3.5, the set $A_{1} \cap F_{2}$ is a stratum of $\partial A_{1}$ and we have that $d_{A_{1} \cap F_{2}}(p)=f_{2}(p)=a_{2}$. On the other hand, if $q \in A_{1}$ is a point at maximal distance to $A_{1} \cap F_{2}$, it follows that $a_{2} \geq d_{A_{1} \cap F_{2}}(q) \geq d_{A_{1} \cap F_{2}}(p)=a_{2}$. This implies that $q \in A_{1} \cap A_{2}$. Therefore, the set $A:=A_{1} \cap A_{2}$ coincides with the set of points in $A_{1}$ at maximal distance to the boundary stratum $A_{1} \cap F_{2}$. Analogously, $A$ coincides with the set of points in $A_{2}$ at maximal distance to $A_{2} \cap F_{1}$. By induction assumption, $A_{1}$ and $A_{2}$ are products with souls as first factors. Thus, a product structure is induced on $A$ in a priori two different ways. They coincide if the first factors coincide. In other words, if there exists at least one soul in $A$ which is a soul of both supersets $A_{1}$ and $A_{2}$, then this holds for all souls of $A$. Let $S \subseteq A$ be a soul of $A_{1}$ given as the intersection of boundary strata of $A_{1}$. Then $S$ is in fact given as the intersection of primitive boundary strata of $A_{1}$ and hence of $A$. This follows easily from the Splitting Theorem, compare also [31, Corollaries 4.1, 4.2]. Since the stratification of $\partial A$ into primitive strata is unique, $S$ is also the intersection of primitive boundary strata of $A_{2}$. Therefore, $S$ is also a soul of $A_{2}$.

7.4 Proposition All souls of the form $S_{i}:=F_{1} \cap \ldots \cap \widehat{F}_{i} \cap \ldots \cap F_{k+1}$ are isometric for $i=1, \ldots, k+1$.

Proof The assertion will be proved for the souls $S_{1}$ and $S_{k+1}$. At first we assume that there is $T \in(0, \infty)$ such that $\Phi_{f_{k+1}}^{T}\left(S_{1}\right) \subseteq S_{k+1}$ and $\Phi_{f_{1}}^{T}\left(S_{k+1}\right) \subseteq S_{1}$. Then the map $\Phi:=\Phi_{f_{1}}^{T} \circ \Phi_{f_{k+1}}^{T}: S_{1} \rightarrow S_{1}$ is nonexpanding. We claim that $\Phi$ is homotopic to the identity on $S_{1}$. Indeed, for $t \in[0,1)$ we set $M_{t}:=f_{1}^{-1}\left(\left[t a_{1}, a_{1}\right]\right)$ and define 
a map $\Phi_{t}$ analogously to $\Phi$ from above, but in the space $M_{t}$. It is clear that this works while keeping the value of $T$. We have that $\Phi_{0}=\Phi$ and in addition we set $\Phi_{1}:=\mathrm{id}_{S_{1}}$. In order to show that $(t, p) \mapsto \Phi_{t}(p)$ is a homotopy, it is sufficient to show that $t \mapsto \Phi_{t}(p)$ is continuous for each fixed $p \in S_{1}$. This follows because all maps $\Phi_{t}$ are nonexpanding. The continuity of $t \mapsto \Phi_{t}(p)$ is a consequence of the fact that $s \mapsto \Phi_{f_{k+1}}^{s}$ is continuous and $f_{k+1}$ stays the same in all superlevel sets $M_{t}$. In addition, $\Phi_{f_{1}}$ is nonexpanding and $f_{1}$ changes only by an additive constant in the superlevel sets $M_{t}$. Hence the claim is proved.

We show now that $\Phi$ is an isometry. By Proposition 7.1, the soul $S_{1}$ is an Alexandrov space of dimension $n-k$ without boundary. According to Grove and Petersen [6, Lemma 1], the $(n-k)$ th Alexander-Spanier cohomology of $S_{1}$ is non-trivial, that is, $\bar{H}^{n-k}\left(S_{1}, \mathbb{Z}_{2}\right)=\mathbb{Z}_{2}$. On the other hand, for each $p \in S_{1}$ we have that $\bar{H}^{n-k}\left(S_{1} \backslash\{p\}, \mathbb{Z}_{2}\right)=0$. Indeed, using the gradient flow of the function $d_{p}^{2}$, we get that $S_{1} \backslash\{p\}$ is homotopically equivalent to some space of dimension less than $n-k$. Therefore, the map $\Phi$ is surjective, because the homotopy $\Phi \simeq \operatorname{id}_{S_{1}}$ implies that the induced homomorphism $\bar{H}^{n-k}(\Phi): \mathbb{Z}_{2} \rightarrow \mathbb{Z}_{2}$ is an isomorphism. Being a surjective nonexpanding map of a compact metric space onto itself, $\Phi$ is an isometry (see for example Burago-Burago-Ivanov [2, Theorem 1.6.15]).

It may happen, however, that such finite $T$ does not exist. In this case we use the fact that by induction assumption all souls of a maximum set $A_{i}$ are isometric. If $A_{1}$ and $A_{k+1}$ intersect, take some $p \in A_{1} \cap A_{k+1}$ and consider the soul $S(p)$. According to Proposition 7.1, the souls $S_{1}$ and $S_{k+1}$ are also souls of the spaces $A_{1}$ and $A_{k+1}$, respectively, and $S(p)$ is a soul of both spaces. This implies that $S_{1} \cong S(p) \cong S_{k+1}$ as desired. Thus, we may assume that $A_{1} \cap A_{k+1}=\varnothing$. Choose a point $p \in\left(F_{2} \cap\right.$ $\left.\ldots \cap F_{k}\right) \backslash\left(A_{1} \cup A_{k+1}\right)$ and consider the superlevel set $M^{\prime}:=f_{1}^{-1}\left(\left[f_{1}(p), a_{1}\right]\right)$. Then there exists some $T \in(0, \infty)$ such that $\Phi_{f_{k+1}}^{T}\left(S_{1}\right) \subseteq S_{k+1}^{\prime}:=F_{1}^{\prime} \cap \ldots \cap F_{k}^{\prime}$. Indeed, since $S_{1}$ is compact, such $T$ exists for each finite $\varepsilon$-net $N$ of $S_{1}$, and since the gradient flow is nonexpanding, all other points are pushed $\varepsilon$-close to $\Phi_{f_{k+1}}^{T}(N)$. It is clear by construction that $A_{1}^{\prime}=A_{1}$ and $A_{1}^{\prime} \cap A_{k+1}^{\prime}=\varnothing$, where $A_{1}^{\prime}, A_{k+1}^{\gamma}$ are defined as $A_{1}, A_{k+1}$, but for the space $M^{\prime}$. Therefore, we can pass to some superlevel set $M^{\prime \prime} \subseteq M^{\prime}$ by shifting the boundary stratum $F_{k+1}^{\prime}$ in an analogous way and obtain that the space $M^{\prime \prime}$ fulfills the assumptions from the first part of the proof. In particular, the corresponding souls $S_{1}^{\prime \prime}$ and $S_{k+1}^{\prime \prime}$ of $M^{\prime \prime}$ are isometric. This in turn holds if we choose $p$ from above arbitrarily close to $A_{k+1}$. By Lemma 4.1, the particular souls $S_{k+1}^{\prime \prime}$ converge to a soul of $A_{k+1}$. Since all souls of $A_{k+1}$ are isometric, we have that $S_{k+1}^{\prime \prime} \cong S_{k+1}$. The same construction applied to $S_{1}^{\prime \prime}$ gives that $S_{1} \cong S_{1}^{\prime \prime}$ and therefore $S_{1} \cong S_{k+1}$. 


\subsection{Corollary All souls of $M$ are isometric.}

Proof Let $S \subseteq M$ be a soul. It is sufficient to prove that $S \cong S_{1}:=F_{2} \cap \ldots \cap F_{k+1}$. If $S \subseteq A_{1}$, the assertion follows by induction assumption. Therefore, we may assume that $s_{1}:=f_{1}(S)<a_{1}$ and consider the superlevel set $M^{\prime}:=f_{1}^{-1}\left(\left[s_{1}, a_{1}\right]\right)$. Proposition 7.4 implies that $S_{1} \cong F_{1}^{\prime} \cap F_{3}^{\prime} \cap \ldots \cap F_{k+1}^{\prime}$. If $S \subseteq A_{2}^{\prime}$, the assertion is proved; otherwise iterate the process of passing to superlevel sets.

These results can be improved further in order to obtain that any soul of $M$ can be "shifted" continuously onto any other soul.

7.6 Lemma Let $S, S^{\prime}$ be souls of $M$. Then there exists a continuous map $\Upsilon: S \times$ $[0,1] \rightarrow M$ such that $\Upsilon^{\tau}(S):=\Upsilon(S, \tau)$ is a soul for each $\tau \in[0,1]$ and $\Upsilon^{0}(S)=$ $S, \Upsilon^{1}(S)=S^{\prime}$.

Proof The proof of Corollary 7.5 shows that it is sufficient to prove the assertion for the case that $S$ and $S^{\prime}$ are given as intersections of $k$ boundary strata. This case, in turn, is treated like in the proof of Proposition 7.4. We define $S_{1}$ and $S_{k+1}$ like there. Again, we first assume that there exists some finite time $T$ such that $\Phi_{f_{k+1}}^{T}\left(S_{1}\right) \subseteq S_{k+1}$. In fact, Proposition 7.4 implies equality, since $\Phi_{f_{k+1}}^{T}$ maps $S_{1}$ isometrically. We write $\Phi:=\Phi_{f_{k+1}}^{T}$ for short and consider it as a map $\Phi: F_{2} \cap \ldots \cap F_{k} \rightarrow S_{k+1}$. Consequently, $\Phi$ maps each soul in $F_{2} \cap \ldots \cap F_{k}$ isometrically onto $S_{k+1}$. If we show that also the sets $\Phi_{f_{k+1}}^{t}\left(S_{1}\right)$ are souls for all $t \in[0, T]$, the desired map $\Upsilon$ is given by $\Phi_{f_{k+1}}$ (after reparametrizing $[0, T] \rightarrow[0,1]$ ).

Let $p, q \in S_{1}$ be distinct points and let $P:=\Phi^{-1}(\Phi(p)), Q:=\Phi^{-1}(\Phi(q))$ denote the fibers through $p$ and $q$, respectively. We claim the following: $P$ and $Q$ are equidistant with $|P Q|=|p q|$, and points $x \in P, y \in Q$ fulfilling $|x y|=|P Q|$ have to lie in the same soul $S(x)=S(y)$. In order to prove the claim, let $\gamma$ be some shortest path from $P$ to $Q$. Since $\Phi$ is 1 -Lipschitz, we obtain that length $(\Phi(\gamma)) \leq \operatorname{length}(\gamma)$ and therefore equality. Moreover, the fact that $\left.\Phi\right|_{S_{1}}: S_{1} \rightarrow S_{k+1}$ is an isometry implies that $|p q|=$ length $(\Phi(\gamma))=$ length $(\gamma)=|P Q|$. For the equidistance let $x \in P$ and $y:=S(x) \cap Q$, the latter being well-defined since $\left.\Phi\right|_{S(x)}$ is an isometry. This clearly also implies that $|x y|=|P Q|$. By swapping the roles of $x$ and $y$, the equidistance of $P$ and $Q$ is proved. Now assume, by way of contradiction, there is $z \in Q$ satisfying $|x z|=|x y|$ and $z \notin S(x)=S(y)$. Then there is some $s \in x z$ such that $s \notin S(x) \cup S(z)$. Let $u:=S(s) \cap Q$. This implies, according to the results above, that $|s u| \leq|s z|$. Hence, we have that

$$
|x z|=|x s|+|s z| \geq|x s|+|s u| \geq|x u| \geq|x Q|=|x y|=|x z|
$$


and therefore everywhere equality. But this means that the shortest paths $x u$ and $x z$ branch at the point $s$, which is a contradiction. Now let $t \geq 0$. Since $\left.\Phi\right|_{S_{1}}$ is an isometry and $\Phi_{f_{k+1}}^{t}$ is 1-Lipschitz, the restriction $\left.\Phi_{f_{k+1}}^{t}\right|_{S_{1}}$ is also an isometry onto its image. This implies that $\left|\Phi_{f_{1}}^{t}(p) \Phi_{f_{1}}^{t}(q)\right|=|p q|$, and by the claim the points $\Phi_{f_{k+1}}^{t}(p)$ and $\Phi_{f_{k+1}}^{t}(q)$ lie in the same soul.

If a finite $T$ like above does not exist, the flow $\Phi_{f_{k+1}}^{t}$ can be extended to $t=\infty$ via Hausdorff limits. This also works like in the proof of Proposition 7.4. By reparametrization $[0, \infty] \rightarrow[0,1]$, we obtain the desired map $\Upsilon$.

\section{Submetries and Retractions}

We will now prove that the term "soul" is fully justified. The respective result is the key point in the proof of our Splitting Theorem. First, we recall the definition of submetries.

8.1 Definition Let $X$ and $Y$ be metric spaces. A map $f: X \rightarrow Y$ is a submetry if $f\left(\bar{B}_{r}(x)\right)=\bar{B}_{r}(f(x))$ holds for all $x \in X$ and all $r \geq 0$. Here, $\bar{B}_{r}(x)$ denotes the closed ball of radius $r$ centered at $x$.

8.2 Proposition For each soul of the form $S_{j}:=F_{1} \cap \ldots \cap \widehat{F}_{j} \cap \ldots \cap F_{k+1}, j \in$ $\{1, \ldots, k+1\}$ there exists a submetry $\Psi_{j}: M \rightarrow S_{j}$ such that the following holds: If $S \subseteq M$ is a soul, the restricted map $\left.\Psi_{j}\right|_{S}: S \rightarrow S_{j}$ is an isometry.

Moreover, $\Psi_{j}$ is composed of gradient flows like follows: $\Phi_{f_{j}}^{T}$ is a submetry of $M$ onto $A_{j}$, possibly with $T=\infty$. The latter case is defined via (possibly not uniquely determined) limits. If $S_{j} \subsetneq A_{j}$, the flowing process is iterated inside $A_{j}$ and hence, $\Psi_{j}$ is obtained as the composition of these gradient flows. In addition, if $S \subseteq M$ is a soul and $t \in[0, \infty]$, the set $\Phi_{f_{j}}^{t}(S)$ is also a soul of $M$.

Proof The proof is carried out for $j=1$, that is, for $\Psi_{1}: M \rightarrow S_{1}$. Let $S \subseteq M$ be a soul. First we consider the case that there is some finite $T \geq 0$ such that $\Phi_{f_{1}}^{T}(M)=S_{1}$. According to Lemma 7.6, there is a continuous map $\Upsilon: S_{1} \times[0,1] \rightarrow M$ such that $\Upsilon^{0}\left(S_{1}\right)=S_{1}$ and $\Upsilon^{1}\left(S_{1}\right)=S$. Thus, the composition $\Phi_{f_{1}}^{T} \circ \Upsilon^{1}: S_{1} \rightarrow S_{1}$ is homotopic to $\Phi_{f_{1}}^{T} \circ \Upsilon^{0}=\operatorname{id}_{S_{1}}$. Like in the proof of Proposition 7.4, the argument involving Alexander-Spanier cohomology shows that $\left.\Phi_{f_{1}}\right|_{S}: S \rightarrow S_{1}$ is an isometry. Moreover, since this holds for any soul $S$, we can repeat the argument from the proof of Lemma 7.6 in order to see that the sets $\Phi_{f_{1}}^{t}(S)$ are souls for all $t \geq 0$.

If a finite $T$ only exist such that $\Phi_{f_{1}}^{T}(M)=A_{1}$, we can use the fact that by induction assumption the desired submetry exists inside $A_{1}$. Let $\Psi_{1}^{A_{1}}$ denote this submetry. 
Then we define $\Psi_{1}$ as the composition $\Psi_{1}:=\Psi_{1}^{A_{1}} \circ \Phi_{f_{1}}^{T}$. If even such finite $T$ does not exist, we have to construct a 1-Lipschitz map $\Phi_{f_{1}}^{\infty}: M \rightarrow A_{1}$. Note that this map will not extend the flow $\Phi_{f_{1}}^{t}$ continuously in the $t$-parameter! Let $\left(p_{\ell}\right)_{\ell \in \mathbb{N}} \subseteq M$ be a dense sequence of points and $0 \leq t_{0,1}<t_{0,2}<t_{0,3}<\ldots$ a diverging sequence in $\mathbb{R}$. Since $M$ is compact, the sequence $\left(\Phi_{f_{1}}^{t_{0, i}}\left(p_{1}\right)\right)$ has some partial $\operatorname{limit}_{i \rightarrow \infty}$ denoted by $\Phi_{f_{1}}^{\infty}\left(p_{1}\right)$. Let $\left(t_{1, i}\right)$ be a subsequence of $\left(t_{0, i}\right)$ such that $\Phi_{f_{1}}^{t_{1, i}}\left(p_{1}\right) \stackrel{\rightarrow \rightarrow \infty}{\longrightarrow} \Phi_{f_{1}}^{\infty}\left(p_{1}\right)$. Now define $\Phi_{f_{1}}^{\infty}\left(p_{2}\right)$ as some partial limit of $\left(\Phi_{f_{1}}^{t_{1, i}}\left(p_{2}\right)\right)$, and so on. It is clear by continuity that $\Phi_{f_{1}}^{\infty}\left(p_{\ell}\right) \in A_{1}$ for all $\ell \in \mathbb{N}$. We claim that $\Phi_{f_{1}}^{\infty}:\left\{p_{\ell} \mid \ell \in \mathbb{N}\right\} \rightarrow A_{1}$ is nonexpanding. Indeed, assume that, say, $\left|p_{1} p_{2}\right|=d$ and $\left|\Phi_{f_{1}}^{\infty}\left(p_{1}\right) \Phi_{f_{1}}^{\infty}\left(p_{2}\right)\right|>d$. Then there exists $t_{2, i}$ for $i$ big enough such that

$$
\left|\Phi_{f_{1}}^{t_{2, i}}\left(p_{1}\right) \Phi_{f_{1}}^{t_{2, i}}\left(p_{2}\right)\right|>d
$$

a contradiction. Now, since $A_{1}$ is compact, in particular complete, there exists a (unique) nonexpanding extension $\Phi_{f_{1}}^{\infty}: M \rightarrow A_{1}$. All the arguments of the first part of the proof carry over, if we substitute $\Phi_{f_{1}}^{T}$ by $\Psi_{1}^{A_{1}} \circ \Phi_{f_{1}}^{\infty}$. In particular, $\left.\Phi_{f_{1}}^{\infty}\right|_{S}$ is an isometry onto its image for each soul $S$. This holds independently of the choice of our dense sequence $\left(p_{\ell}\right)$. Therefore, we can conclude that also $\left.\Phi_{f_{1}}^{t}\right|_{S}$ is an isometry onto its image for all $t \geq 0$. Indeed, assume there are points $x, y \in S$ whose distance is decreased by $\left.\Phi_{f_{1}}^{t}\right|_{S}$. Then we substitute $p_{1}, p_{2}$ by $x, y$ and obtain for the corresponding construction of $\Phi_{f_{1}}^{\infty}$ that $\left|\Phi_{f_{1}}^{\infty}(x) \Phi_{f_{1}}^{\infty}(y)\right|<|x y|$, contradiction. Finally, by these results, also the fact that $\Phi_{f_{1}}^{t}(S)$ is a soul for each $t \in[0, \infty]$ and each soul $S$ carries over.

The following consequences are immediate.

8.3 Corollary For each $j \in\{1, \ldots, k+1\}$ the soul $S_{j}=F_{1} \cap \ldots \cap \widehat{F}_{j} \cap \ldots \cap F_{k+1}$ is a strong deformation retract of $M$. Moreover, if we set $D_{j}(p):=\Psi_{j}^{-1}\left(\Psi_{j}(p)\right)$ for $p \in M$, the map $p \mapsto\left(S(p), D_{j}(p)\right)$ is a bijection between $M$ and the set $\left\{\left(S, D_{j}\right) \mid S\right.$ soul of $M, D_{j}$ fiber of $\left.\Psi_{j}\right\}$. The fibers $D_{j}(p), D_{j}(q)$ are equidistant for all $p, q \in M$, and $x \in D_{j}(p), y \in D_{j}(q)$ satisfy $|x y|=\left|D_{j}(p) D_{j}(q)\right|$ if and only if $S(x)=S(y)$.

8.4 Definition For each $j \in\{1, \ldots, k+1\}$ the set $D_{j}(p)$ defined as in Corollary 8.3 is called the $j$ th dual fiber through $p \in M$.

The name indicates that these dual fibers will provide the second factor in our metric splitting of $M$. First, we have to prove that all $j$ th dual fibers through any point actually coincide. 


\section{The dual fibers}

We investigate the canonical projections along souls. They turn out to be locally Lipschitz almost everywhere, which implies that Lipschitz paths are mapped onto Lipschitz paths. Moreover, Rademacher's Theorem can be applied. For that reason, the following result plays a crucial role.

9.1 Proposition Let $j \in\{1, \ldots, k+1\}$ and let $D_{j}$ be a $j$ th dual fiber. Let $\pi_{D_{j}}: M \rightarrow$ $D_{j}$ denote the projection along souls onto $D_{j}$, that is $\pi_{D_{j}}(p)=S(p) \cap D_{j}$ for $p \in M$. Then $\pi_{D_{j}}$ is locally a Lipschitz map near almost all points. More precisely, if $p \in M$ is a regular point of the Alexandrov space $S(p)$, there is an open neighborhood $U \subseteq \stackrel{\circ}{\subseteq} S(p)$ such that $\left.\pi_{D_{j}}\right|_{\Psi_{j}^{-1}\left(\Psi_{j}(U)\right)}$ is Lipschitz.

Proof Let $p \in M$ be a regular point of its soul $S(p)$. In other words, $p$ is $(n-k, \delta)-$ strained in $S(p)$ for all $\delta>0$, see Burago-Burago-Ivanov [2, Definition 10.8.9]. Choose some small $\delta>0$ and an open neighborhood $V \subseteq \stackrel{\circ}{\subseteq}(p)$ of $p$ small enough such that all points $x \in V$ are also $(n-k, \delta)$-strained with the same points $b_{1}, \ldots, b_{2(n-k)}$ of the strainers. We set $h_{i}(x):=\frac{1}{2}\left|b_{i} x\right|^{2}$ for $i=1, \ldots, 2(n-k), x \in S(p)$. By construction, there is a global constant $C>0$ such that $\left|\nabla_{x} h_{i}\right| \geq C$ for all $x \in V, i=$ $1, \ldots, 2(n-k)$. The functions $h_{i}$ are $1-$ concave, and so are all convex combinations $\sum \beta_{i} h_{i}$ (where $\sum \beta_{i}=1, \beta_{i} \geq 0$ ). If $x \in V$ is regular and all differentials $d_{h_{i}}$ are linear, then $\nabla_{x}\left(\sum \beta_{i} h_{i}\right)=\sum \beta_{i} \nabla_{x} h_{i}$ holds for all convex combinations. By the generalized Rademacher Theorem, see for example Lytchak [8, Proposition 3.8], this is true for almost all points $x \in V$, and since they are $(n-k, \delta)$-strained, the positive constant $C$ from above can be chosen such that $\left|\nabla_{x}\left(\sum \beta_{i} h_{i}\right)\right| \geq C$ holds for almost all $x \in V$ and all convex combinations. Moreover, for all such points $x$ and any direction $\xi \in \Sigma_{x} S(p)$, there exist $\beta_{i} \geq 0, \sum \beta_{i}=1$ such that $\nabla_{x}\left(\sum \beta_{i} h_{i}\right)=\sum \beta_{i} \nabla_{x} h_{i}$ has the given direction $\xi$. We will call such points nice.

Now let $U \subseteq V$ be an open neighborhood of $p$ small enough such that $x y \subseteq V$ for all $x, y \in U$. Moreover, if any two points $x, y \in U$ are given, there is a shortest path $\gamma$ with endpoints in $U$ arbitrarily close to $x$ and $y$, respectively, such that almost all points on $\gamma$ are nice. This fact can be proved by adapting a proof by Otsu and Shioya in [15]. They showed that the property holds if all regular points instead of nice points are considered. The adapted proof can be found in the author's $\mathrm{PhD}$ thesis [31, Proposition 2.1]. It follows that almost every tangent vector of $\gamma$ coincides with the gradient of a suitable convex combination $\sum \beta_{i} h_{i}$. To be precise, the equality of the vectors holds only up to length; we reparametrize $\gamma$ to get full equality. Since all gradient lengths are bounded below by $C$, the reparametrized path $\gamma$ is defined on an 
interval of length at most $T<\infty$. Here, $T$ is a universal constant valid for all such paths $\gamma$, independent of the given points $x, y \in U$. The proof of the Proposition is now carried out in the subsequent three steps.

Step 1 For any $x, y \in U$ the projection along souls from $D_{j}(x)$ onto $D_{j}(y)$ is $e^{T}$-Lipschitz.

In order to show this, we define the functions $\hat{h}_{i}: M \rightarrow \mathbb{R}, q \mapsto \frac{1}{2}\left|q D_{j}\left(b_{i}\right)\right|^{2}$ for $i=1, \ldots, 2(n-k)$. It follows that also the functions $\hat{h}_{i}$ are 1-concave. Furthermore, according to Corollary 8.3, we have that $\left.\widehat{h}_{i}\right|_{S(p)}=h_{i}$. Since all souls are isometric, a respective statement holds in each soul. Now we assume for the moment that $x$ and $y$ can be connected by some shortest path $\gamma$ as above. Thus, almost every tangent vector of $\gamma$ coincides with the gradient of some convex combination $\sum \beta_{i} \hat{h}_{i}$. For any other soul we get the same result, and since the functions $\hat{h}_{i}$ are defined on the entire space $M$, the Lipschitz property of the gradient push according to Petrunin (see Petrunin [24, 1.3.5 and 2.1.4]) implies that the following holds: If $x_{1}, x_{2} \in D_{j}(x)$ and $y_{\ell}=S\left(x_{\ell}\right) \cap D_{j}(y), \ell=1,2$, then $\left|y_{1} y_{2}\right| \leq e^{T}\left|x_{1} x_{2}\right|$. If $x$ and $y$ cannot be connected directly by such path $\gamma$, there are adequate points in each neighborhood of $x$ and $y$. Therefore, the Lipschitz constant stays the same.

Step 2 For any $x \in U$ the projection along souls from $\Psi_{j}^{-1}\left(\Psi_{j}(U)\right)$ onto $D_{j}(x)$ is $2 e^{T}$-Lipschitz.

This follows easily from Step 1 . Indeed, let $q, r \in \Psi_{j}^{-1}\left(\Psi_{j}(U)\right)$ and project $r$ onto $D_{j}(q)$, that is, $r$ is mapped onto $r^{\prime}:=S(r) \cap D_{j}(q)$. We obtain that

$$
\left|q r^{\prime}\right| \leq|q r|+\left|r r^{\prime}\right| \leq|q r|+\left|D_{j}(r) D_{j}(q)\right| \leq 2|q r|
$$

and hence the statement follows by Step 1 .

Step 3 The projection along souls from $\Psi_{j}^{-1}\left(\Psi_{j}(U)\right)$ onto $D_{j}$ is Lipschitz.

For the proof take some shortest path $\tau$ from $p$ to $D_{j}$. In other words, $\tau$ lies in $S(p)$ and ends at $S(p) \cap D_{j}$. Let $z \in \tau$ be an interior point close enough to $p$ such that $z \in U$. According to Step 2, the projection along souls from $\Psi_{j}^{-1}\left(\Psi_{j}(U)\right)$ onto $D_{j}(z)$ is Lipschitz. Hence, it is sufficient to show that also the projection along souls from $D_{j}(z)$ onto $D_{j}$ is Lipschitz. It is clear that the gradient flow of the function $x \mapsto \frac{1}{2}|x p|^{2}$ pushes $z$ along $\tau$ onto $S(p) \cap D_{j}$ and the same is true via isometric copies in all souls. Like in Step 1, the gradient flow of the function $x \mapsto \frac{1}{2}\left|x D_{j}(p)\right|^{2}$ has the identical action on each soul. Moreover, since $z \neq p$, the flow pushes $D_{j}(z)$ onto $D_{j}$ in finite time. Hence, the Lipschitz property of the gradient push implies that the projection along souls is Lipschitz, too. 
9.2 Corollary For any $j$ th dual fibers $D_{j}, D_{j}^{\prime}$ the projection along souls from $D_{j}$ onto $D_{j}^{\prime}$ is a homeomorphism.

Proof Let $p \in M$ be regular in its soul $S(p)$. By Proposition 9.1, the projections $D_{j}(p) \rightarrow D_{j}$ and $D_{j}(p) \rightarrow D_{j}^{\prime}$ are in particular continuous, hence (by compactness and bijectivity) homeomorphisms.

9.3 Remark The fact that points $p \in M$ which are regular in its soul $S(p)$ form a set of full measure in $M$ follows by Proposition 8.2 and Rademacher's Theorem. Indeed, each submetry $\Psi_{j}$ is differentiable almost everywhere with linear differential. The points where this is true are as requested.

Lytchak's results [8] about submetries imply the following statements.

9.4 Lemma For $p \in M$ and $j \in\{1, \ldots, k+1\}$ let $S:=S(p)$ and $D:=D_{j}(p)$. Then the tangent cones $T_{p} S$ and $T_{p} D$ are convex subsets of $T_{p} M$ and therefore Alexandrov spaces. Their Hausdorff dimensions are $\operatorname{dim}_{H} T_{p} S=n-k$ and $\operatorname{dim}_{H} T_{p} D=k$. In addition, we have that $T_{p} S=\left\{u \in T_{p} M \mid\langle u, v\rangle=0 \quad \forall v \in T_{p} D\right\}$.

Proof Since $S \subseteq M$ is a convex subset of Hausdorff dimension $n-k$, the same holds for the tangent cone $T_{p} S$. According to Proposition 8.2, the map $\Psi_{i}: M \rightarrow S_{i}:=F_{1} \cap$ $\ldots \cap \widehat{F}_{i} \cap \ldots \cap F_{k+1}$ is a submetry. By [8, Proposition 5.1] it induces a homogeneous submetry $d_{p} \Psi_{i}: T_{p} M \rightarrow T_{p} S_{i}$ of the tangent cones. Again Proposition 8.2 implies that $\left.d_{p} \Psi_{i}\right|_{T_{p} S}$ is an isometry. Therefore, if $u \in T_{p} S$, then $d_{p} \Psi_{i}$ preserves its length (this property of $u$ is called horizontal by Lytchak). Now [8, Lemma 5.3] and Corollary 8.3 imply that the converse is also true, that is, $u \in T_{p} M$ fulfills $\left|d_{p} \Psi_{i}(u)\right|=|u|$ if and only if $u \in T_{p} S$. Hence, $d_{p} \Psi_{i}$ is a regular submetry as defined in [8, Definition 6.4]. It follows from [8, Korollar 7.5] that $\operatorname{dim}_{H} T_{p} D=k$. The cone $T_{p} D \subseteq T_{p} M$ coincides with the preimage $\left(d_{p} \Psi_{i}\right)^{-1}(o)$ by [8, Proposition 5.2]. The latter is a convex subset by [8, Proposition 6.4(1)], while (2) implies that $d_{p} \Psi_{j}$ preserves the lengths of precisely those vectors in the cone $K(P)$ over the polar set $P \subseteq \Sigma_{p}$ of $\Sigma_{p} D$, that is $P=\left\{\xi \in \Sigma_{p}|| \xi \Sigma_{p} D \mid \geq \frac{\pi}{2}\right\}$. Therefore we obtain that $T_{p} S=K(P)$ and hence $T_{p} S=\left\{u \in T_{p} M \mid\langle u, v\rangle \leq 0 \quad \forall v \in T_{p} D\right\}$.

Now let $q \in S, q \neq p$ and $\xi \in \Sigma_{p} D$. Assume, by way of contradiction, that $\left|\xi \uparrow_{m \rightarrow \infty}^{q}\right|>\frac{\pi}{2}$. We choose in $D$ a sequence $p_{m} \stackrel{m \rightarrow \infty}{\longrightarrow} p$ such that $\uparrow_{p}^{p_{m}} \rightarrow \xi$ and set $q_{m}:=S\left(p_{m}\right) \cap$ $D_{j}(q)$. Corollary 9.2 implies that $q_{m} \rightarrow q$. By passing to a subsequence we may assume that the directions $\uparrow_{q}^{q_{m}}$ converge to some $\zeta \in \Sigma_{q} D_{j}(q)$. Now the assumption together with $|\zeta \uparrow q ~ p| \geq \frac{\pi}{2}$ (by the previous result) implies that there exists some $N \in \mathbb{N}$ such that $\left|p_{N} q_{N}\right|>|p q|$. This contradicts Corollary 8.3. 
By lower semi-continuity of angles the result holds for all directions in $\Sigma_{p} S$. In other words, we obtain that $\langle u, v\rangle=0$ for all $u \in T_{p} S$ and all $v \in T_{p} D$. Thus, the Lemma is proved.

We are now able to prove that we do not have to distinguish $j$ th dual fibers, but there are unique dual fibers through all points.

9.5 Proposition For all $i, j \in\{1, \ldots, k+1\}$ and any $p \in M$ we have that $D_{i}(p)=$ $D_{j}(p)=: D(p)$

Proof The assertion is proved for $i=1, j=2$ and almost all points $p$. Let $p \in M$ such that it is regular in the soul $S(p)$. Let $S \subseteq M$ be a soul and set $q_{1}:=S \cap D_{1}(p), q_{2}:=S \cap D_{2}(p)$. Assume, by way of contradiction, that $q_{1} \neq q_{2}$. Since all dual fibers are path connected, $q_{1}$ can be assumed to lie arbitrarily close to $p$. Moreover, by [8, Theorem 7.2] the induced metric and the induced intrinsic metric on dual fibers are locally bi-Lipschitz equivalent. Thus, there exists a Lipschitz path $\gamma_{1}:[a, b] \rightarrow D_{1}(p)$ from $p$ to $q_{1}$. By choosing $q_{1}$ close enough to $p$, we can apply Proposition 9.1 and obtain that the projection along souls onto $D_{2}(p)$ maps $\gamma_{1}$ onto a Lipschitz path $\gamma_{2} \subseteq D_{2}(p)$ from $p$ to $q_{2}$.

According to Perelman and Petrunin [21, Proposition 2.1(a)], the left and right tangent vectors $\gamma_{\ell}^{+}(t), \gamma_{\ell}^{-}(t)$ exist and are opposite for almost all $t \in[a, b], \ell=1,2$. Thus, the function $L:[a, b] \rightarrow \mathbb{R}, t \mapsto\left|\gamma_{1}(t) \gamma_{2}(t)\right|$ is differentiable for almost all $t$. Moreover, for such $t$ we have that $L^{\prime}(t)=0$ by Lemma 9.4. Since $L$ is Lipschitz, in particular absolutely continuous, $L$ is constant. This implies that $0 \neq\left|q_{1} q_{2}\right|=L(a)=L(b)=$ $|p p|=0$, contradiction.

Thus, the Theorem is proved for the dual fibers through almost all points $p \in M$. The equality of $i$ th and $j$ th dual fibers carries over to their Hausdorff limits (being also $i$ th and $j$ th dual fibers, respectively, by Proposition 8.2) and hence, the statement is proved for all dual fibers.

We are now prepared for proving that the souls form equidistant subsets.

\section{Equidistance of the souls}

The previous results already imply that certain souls are equidistant, namely along shortest paths to the boundary strata $F_{i}$. More precisely, we obtain the following result. 
10.1 Proposition Let $i \in\{1, \ldots, k+1\}$ and denote by $\alpha_{x}$ the gradient curve of $f_{i}$ starting at $x \in M$. Then for each soul $S \subseteq M$ and $t \geq 0$ we have that

$$
\operatorname{length}\left(\left.\alpha_{p}\right|_{[0, t]}\right)=\operatorname{length}\left(\left.\alpha_{q}\right|_{[0, t]}\right) \quad \forall p, q \in S .
$$

Proof The proof is carried out for $i=1$. Let $p, q \in S$ and let $\gamma$ be some shortest path from $p$ to $q$. Choose two distinct interior points $p_{0}, q_{0} \in \gamma$. We claim that $\left|\nabla_{p_{0}}\left(f_{1}\right)\right|=\left|\nabla_{q_{0}}\left(f_{1}\right)\right|$.

If $S \subseteq A_{1}$, there is nothing to prove. Thus, we may assume that $S \subseteq F_{1}$, because otherwise we could proceed in the superlevel set $f_{1}^{-1}\left(\left[f_{1}(S), a_{1}\right)\right]$. If there is some $j \in\{2, \ldots, k+1\}$ with $S \subseteq F_{j}$, the direction $\xi$ of the gradient $\nabla_{p_{0}}\left(f_{1}\right)$ satisfies $\xi \in \Sigma_{p_{0}} F_{j}$. This implies that $\nabla_{p_{0}}\left(f_{1}\right)$ stays the same if we consider the doubling $\bar{M}$ obtained by gluing along $F_{2} \cup \ldots \cup F_{k+1}$. In $\bar{M}$ we have that $\bar{\Sigma}_{p_{0}} \bar{F}_{1}=\partial \bar{\Sigma}_{p_{0}}$. The direction $\xi$ is the (unique) one at maximal distance to $\partial \bar{\Sigma}_{p_{0}}$. The analog statement holds for $q_{0}$ and since $p_{0}, q_{0}$ are interior points of $\gamma$, Petrunin's parallel transportation implies that $\bar{\Sigma}_{p_{0}} \cong \bar{\Sigma}_{q_{0}}$, see [23, Theorem 1.1A]. Hence, the claim $\left|\nabla_{p_{0}}\left(f_{1}\right)\right|=\left|\nabla_{q_{0}}\left(f_{1}\right)\right|$ follows.

Now let $t \geq 0$. According to Proposition 8.2, the pushed soul $\Phi_{f_{1}}^{t}(S)$ is again a soul of $M$ and is isometric to $S$ via the gradient flow. In particular, $\Phi_{f_{1}}^{t}(\gamma)$ is a shortest path from $\Phi_{f_{1}}^{t}(p)$ to $\Phi_{f_{1}}^{t}(q)$ containing the distinct interior points $\Phi_{f_{1}}^{t}\left(p_{0}\right), \Phi_{f_{1}}^{t}\left(q_{0}\right)$. For these points the claim from above holds, too. This implies that the gradient curves starting at $p_{0}$ and $q_{0}$, respectively, satisfy length $\left(\left.\alpha_{p_{0}}\right|_{[0, t]}\right)=\operatorname{length}\left(\left.\alpha_{q_{0}}\right|_{[0, t]}\right)$.

We choose now sequences $\left(p_{n}\right),\left(q_{n}\right)$ of interior points of $\gamma$ such that $p_{n} \rightarrow p$ and $q_{n} \rightarrow q$. The argument from above carries over to all $p_{n}, q_{n}$, and we obtain sequences of converging gradient curves with equal lengths. According to Petrunin [24, Lemma 2.1.5] and its proof, the limits are the gradient curves $\alpha_{p}$ and $\alpha_{q}$, respectively, having the same lengths on each subinterval.

10.2 Corollary For each $i \in\{1, \ldots, k+1\}$ the following holds: Let $p \in M \backslash F_{i}$ and let $\gamma$ be some shortest path from $p$ to $F_{i}$. Then for any dual fiber $D$ the canonical projection $\gamma \rightarrow D, \gamma(t) \mapsto S(\gamma(t)) \cap D$ is a shortest path from $q:=S(p) \cap D$ to $F_{i}$.

Proof Let $p_{0} \in F_{i}$ be the endpoint of $\gamma$ and set $q_{0}:=S\left(p_{0}\right) \cap D$. We denote by $\gamma^{\leftarrow}:\left[0, f_{i}(p)\right] \rightarrow M, t \mapsto \gamma\left(f_{i}(p)-t\right)$ the curve coinciding with $\gamma$, but with reversed parametrization. Then the (unit speed) curve $\gamma^{\leftarrow}$ coincides with the gradient curve $\left.\alpha_{p_{0}}\right|_{\left[0, f_{i}(p)\right]}$ of the function $f_{i}$. According to Proposition 10.1, it has the same length as the gradient curve $\left.\alpha_{q_{0}}\right|_{\left[0, f_{i}(p)\right]}=: \delta$. Hence, $\delta^{\leftarrow}$ is a shortest path from $\delta\left(f_{i}(p)\right)$ to $F_{i}$. Finally, by Proposition 8.2, the curve $\delta \leftarrow$ coincides with the canonical projection of $\gamma$ along souls and $\delta\left(f_{i}(p)\right)=q$. 
In order to show in the end that all souls are equidistant subsets of $M$, we will use the projection along souls to transport some shortest path between two souls into any dual fiber. The key point is to show that such projections are 1-Lipschitz maps. This in turn will be proved using the differential near souls with special properties.

10.3 Lemma Let $p \in M \backslash \partial M$ such that $\left|\Uparrow_{p}^{F_{i}}\right|=1$ for all $i \in\{1, \ldots, k+1\}$. Then $\left|\Uparrow_{q}^{F_{i}}\right|=1$ holds also for all $i \in\{1, \ldots, k+1\}$ and all $q \in S(p)$. Moreover, we have that $\left|\uparrow F_{q} \uparrow_{q}^{F_{j}}\right|=\left|\uparrow F_{p} \uparrow_{p}^{F_{j}}\right|$ for all $i, j \in\{1, \ldots, k+1\}$.

Proof Choose some $q \in S(p)$. The first statement is an immediate consequence of Corollary 10.2. This Corollary also implies, together with Proposition 9.5, that the shortest path from $q$ to any $F_{i}$ coincides with the projection along souls of the shortest path from $p$ to $F_{i}$ into the unique dual fiber $D(q)$. Therefore, the directional derivative $d_{p} f_{i}\left(\uparrow_{p} F_{j}\right)$ coincides with $d_{q} f_{i}\left(\uparrow F_{q}\right)$ for all $i, j \in\{1, \ldots, k+1\}$. This implies the second statement.

The following is just a technical lemma.

10.4 Lemma Let $1 \leq \ell \leq k$ and $v_{1}, \ldots, v_{\ell+1} \in \mathbb{R}^{k}$ such that the following holds:

(1) $v_{1}, \ldots, v_{\ell+1}$ are minimal linearly dependent, that is, the vectors of any subcollection $v_{1}, \ldots, \widehat{v}_{i}, \ldots, v_{\ell+1}$ are linearly independent for all $i \in\{1, \ldots, \ell+1\}$;

(2) $\measuredangle\left(v_{i}, v_{j}\right) \geq \frac{\pi}{2} \quad \forall i \neq j$.

Let $V:=\operatorname{span}\left(v_{1}, \ldots, v_{\ell+1}\right)$ and $W:=\left\{w \in \mathbb{R}^{k} \mid\left\langle v_{i}, w\right\rangle \leq 0 \forall i \in\{1, \ldots, \ell+1\}\right\}$. Then $W$ is a vector subspace fulfilling $\operatorname{dim} W=k-\ell$ and $W=V^{\perp}$.

Proof The main part is to prove that $V=\left\{\sum_{i=1}^{\ell+1} \alpha_{i} v_{i} \mid \alpha_{i} \geq 0\right\}$. We will use induction over $\ell$. The base step $\ell=1$ is clear, hence let $\ell \geq 2$. In the following we work in $V=\mathbb{R}^{\ell}$ and we assume without loss of generality that $v_{\ell+1}=(-1,0, \ldots, 0)$. For $i=1, \ldots, \ell$ we define $w_{i}$ as the orthogonal projection of $v_{i}$ onto $v_{\ell+1}^{\perp}$, that is, $w_{i}$ is obtained by setting the first component of $v_{i}$ to 0 . According to our assumptions, $w_{i} \neq 0$ for $i=1, \ldots, \ell$. We claim that $w_{1}, \ldots, w_{\ell} \in v_{\ell+1}^{\perp} \cong \mathbb{R}^{\ell-1}$ fulfill the induction hypothesis.

It is clear that $w_{1}, \ldots, w_{\ell}$ are linearly dependent. Now assume that, say, $w_{2}, \ldots$, $w_{\ell}$ are already linearly dependent. Then there is a non-trivial linear combination $\sum_{i=2}^{\ell} \lambda_{i} w_{i}=0$. By definition of the $w_{i}$, this implies that $\sum_{i=2}^{\ell} \lambda_{i} v_{i}=\lambda v_{\ell+1}$ for some $\lambda \in \mathbb{R}$. Thus, $v_{2}, \ldots, v_{\ell+1}$ are linearly dependent, which contradicts the assumptions. In addition we have that $\measuredangle\left(w_{i}, w_{j}\right) \geq \frac{\pi}{2}$ for all $i \neq j$. Indeed, for each 
$i=1, \ldots, \ell$ the unit vectors $\frac{v_{i}}{\left|v_{i}\right|}, \frac{v_{\ell+1}}{\left|v_{\ell+1}\right|} \in \mathbb{S}^{\ell-1}$ can be connected by a unique shortest path in $\mathbb{S}^{\ell-1}$. Its intersection point with $v_{\ell+1}^{\perp}$ coincides with the unit vector $\frac{w_{i}}{\left|w_{i}\right|}$. Now it is easy to see that $\measuredangle\left(w_{i}, w_{j}\right)<\frac{\pi}{2}$ would imply that $\measuredangle\left(v_{i}, v_{j}\right)<\frac{\pi}{2}$.

Hence, by induction assumption, we have that $v_{\ell+1}^{\perp}=\left\{\sum_{i=1}^{\ell} \alpha_{i} w_{i} \mid \alpha_{i} \geq 0\right\}$. It follows immediately that the half space $\left\{\left(x_{1}, \ldots, x_{\ell}\right) \in \mathbb{R}^{\ell} \mid x_{1} \leq 0\right\}$ is contained in the convex set $\left\{\sum_{i=1}^{\ell+1} \alpha_{i} v_{i} \mid \alpha_{i} \geq 0\right\}$. Thus, the entire space $V$ is contained if, and only if, there is some $v_{i} \in\left\{\left(x_{1}, \ldots, x_{\ell}\right) \in \mathbb{R}^{\ell} \mid x_{1}>0\right\}$. This is equivalent to $w_{i} \neq v_{i}$. But the latter is satisfied for at least one index $i$, since otherwise $v_{1}, \ldots, v_{\ell}$ would be linearly dependent.

Now we prove the assertion of the Lemma. Let $w \in W$ and $j \in\{1, \ldots, \ell+1\}$. By the previous result, there are coefficients $\alpha_{1}, \ldots, \alpha_{\ell+1} \geq 0$ such that $-v_{j}=\sum_{i=1}^{\ell+1} \alpha_{i} v_{i}$. It follows that

$$
-\left\langle v_{j}, w\right\rangle=\left\langle\sum_{i=1}^{\ell+1} \alpha_{i} v_{i}, w\right\rangle=\sum_{i=1}^{\ell+1} \alpha_{i}\left\langle v_{i}, w\right\rangle \leq 0
$$

and therefore $\left\langle v_{j}, w\right\rangle=0$. We obtain that

$$
W=\left\{w \in \mathbb{R}^{k} \mid\left\langle v_{i}, w\right\rangle=0 \quad \forall i \in\{1, \ldots, \ell+1\}\right\}
$$

which yields the desired results.

We are now able to investigate the differential of the projection along souls almost everywhere.

10.5 Proposition Let $D$ be a dual fiber and $\pi_{D}: M \rightarrow D, x \mapsto S(x) \cap D$ the projection along souls onto $D$. Then for almost all $p \in M$ the differential $d_{p} \pi_{D}$ exists and is 1-Lipschitz.

Proof According to Proposition 9.1, the projection $\pi_{D}$ is locally Lipschitz near almost all points. Hence, it is differentiable almost everywhere with linear differential. More precisely, we consider $\pi_{D}$ as a map into $M$ (thus, the target space is Alexandrov) with image in $D$, where $D$ is equipped with the induced metric. Then for almost all $p \in M$ we have the following.

- $T_{p} M$ is isometric to $\mathbb{R}^{n}$.

- $T_{\pi_{D}(p)} M$ is isometric to $\mathbb{R}^{m} \times C$, where $C \in \operatorname{ALEX}^{n-m}(0)$ is a cone.

- $d_{p} \pi_{D}$ is a linear map with image in the $\mathbb{R}^{m}$-factor of $T_{\pi_{D}(p)} M$. 
Moreover, the Euclidean cone $T_{p} M$ can be assumed to split into the factors $T_{p} S(p) \cong$ $\mathbb{R}^{n-k}$ and $T_{p} D(p) \cong \mathbb{R}^{k}$. Indeed, since to each vector $v \in T_{p} M$ there exists the opposite vector $-v \in T_{p} M$, it follows from Lemma 9.4 that $T_{p} S(p) \cong \mathbb{R}^{n-k}$. According to the proof of that Lemma, $T_{p} D(p)=\left(d_{p} \Psi_{i}\right)^{-1}(0)$ for each $i \in\{1, \ldots, k+1\}$. All maps $\Psi_{i}$ are 1-Lipschitz, hence $d_{p} \Psi_{i}$ is linear at almost all $p \in M$. If we take such a point $p$, it follows that $T_{p} D(p) \cong \mathbb{R}^{k}$.

Finally, we may assume that each shortest path from $p$ to any $F_{i}$ can be extended beyond $p$. Indeed, points where this is not possible form a set of measure zero. Otsu proved in [14] that the cut locus has measure zero. His proof can be adapted to our situation; this has been written up in the author's $\mathrm{PhD}$ thesis [31, Proposition 2.2].

For $i=1, \ldots, k+1$ we set $b_{i}:=\uparrow{ }_{p}^{F_{i}} \in T_{p} D(p)$. It is easy to see that $\left|b_{i} b_{j}\right| \geq \frac{\pi}{2}$ holds for all $i \neq j$. Indeed, this is a consequence of the concavity of $d_{F_{i}}$, compare Lemma 3.5. In addition, Corollary 10.2 and Lemma 10.3 imply that

$$
\left\langle b_{i}, b_{j}\right\rangle=\left\langle d_{p} \pi_{D}\left(b_{i}\right), d_{p} \pi_{D}\left(b_{j}\right)\right\rangle \quad \forall i, j \in\{1, \ldots, k+1\} .
$$

Now let $v \in T_{p} M$. By the orthogonal splitting $T_{p} M=T_{p} S(p) \times T_{p} D(p)$ it is sufficient to consider $v \in T_{p} D(p)$. If there are coefficients $\beta_{i} \in \mathbb{R}$ such that $v=\sum_{i=1}^{k+1} \beta_{i} b_{i}$, then linearity of $d_{p} \pi_{D}$ implies the following.

$$
\begin{aligned}
|v|^{2} & =\langle v, v\rangle \\
& =\sum_{i, j} \beta_{i} \beta_{j}\left\langle b_{i}, b_{j}\right\rangle \\
& =\sum_{i, j} \beta_{i} \beta_{j}\left\langle d_{p} \pi_{D}\left(b_{i}\right), d_{p} \pi_{D}\left(b_{j}\right)\right\rangle \\
& =\left\langle d_{p} \pi_{D}(v), d_{p} \pi_{D}(v)\right\rangle \\
& =\left|d_{p} \pi_{D}(v)\right|^{2}
\end{aligned}
$$

Hence, the Proposition is proved in the case of $\operatorname{span}\left(b_{1}, \ldots, b_{k+1}\right)=T_{p} D(p)$.

In the general case we choose some minimal linearly dependent subcollection of $\left\{b_{i}\right\}$ in the sense of Lemma 10.4. We assume, by renumbering, that $b_{1}, \ldots, b_{\ell+1}$ is such subcollection, where $1 \leq \ell \leq k$. Let $V$ and $W$ be given as in Lemma 10.4 , hence $T_{p} D(p)$ splits orthogonally as $T_{p} D(p)=V \oplus W$. According to the results from above, it is sufficient to consider $v \in W$. For $i=1, \ldots, \ell$ we shift the boundary strata $F_{i}$ to $p$, that is, we consider the intersection $M^{\prime}:=f_{1}^{-1}\left(\left[f_{1}(p), a_{1}\right]\right) \cap \ldots \cap f_{\ell}^{-1}\left(\left[f_{\ell}(p), a_{\ell}\right]\right)$ of superlevel sets. Each boundary stratum $F_{i}$ is perpendicular to the corresponding shortest path from $p$ to $F_{i}$ and the same holds in superlevel sets. It also holds in $M^{\prime}$, 
since all these shortest paths are extendable beyond $p$. Thus, the tangent cone $T_{p} M^{\prime}$ coincides with the set $\left\{v \in T_{p} M \mid\left\langle v, b_{i}\right\rangle \leq 0 \forall i \in\{1, \ldots, \ell\}\right\}$. No collapse occurs, because $b_{1}, \ldots, b_{\ell}$ are linearly independent and therefore $T_{p} M^{\prime}$ has full dimension.

We claim that $\left.f_{\ell+1}\right|_{M^{\prime}}$ attains its maximum at $p \in M^{\prime}$. Indeed, let $w \in T_{p} M^{\prime}$ such that $\left\langle w, b_{\ell+1}\right\rangle \leq 0$. Then $w \in T_{p} S(p) \oplus W$, which implies that in fact $\left\langle w, b_{\ell+1}\right\rangle=0$. The claim follows by concavity of $f_{\ell+1}$. By the claim, the vector $v \in W \subseteq T_{p} M^{\prime}$ can be considered as $v \in W \subseteq T_{p} A_{\ell+1}^{\prime}$, where $A_{\ell+1}^{\prime}=\max _{x \in M^{\prime}} f_{\ell+1}(x)$. More precisely, we have that $T_{p} A_{\ell+1}^{\prime}=T_{p} S(p) \oplus W$. By induction assumption, the statement of the Proposition is proved for the set $A_{\ell+1}^{\prime}$ and hence, $\left|d_{p} \pi_{D}(v)\right|=|v|$. This completes the proof.

10.6 Proposition For each dual fiber $D$, equipped with the induced metric, the projection along souls $\pi_{D}: M \rightarrow D, x \mapsto S(x) \cap D$ is 1-Lipschitz.

Proof Choose an arbitrary dual fiber $D$. Let $p, q \in M$ be distinct points and $\varepsilon>0$. Choose $\delta>0$ small enough such that $\pi_{D}\left(B_{\delta}(x)\right) \subseteq B_{\varepsilon}\left(\pi_{D}(x)\right)$ for $x=p$ and $x=q$ (recall Corollary 9.2). It follows from Proposition 10.5 that there are points $\hat{p} \in B_{\delta}(p), \hat{q} \in B_{\delta}(q)$ such that for almost all points $x \in \hat{p} \hat{q}$ the differential $d_{x} \pi_{D}$ is 1-Lipschitz (see also the remark about nice points in the proof of Proposition 9.1). This implies that $\left|\pi_{D}(\hat{p}) \pi_{D}(\hat{q})\right| \leq|\hat{p} \hat{q}|$. Thus, we obtain the following:

$$
\begin{aligned}
\left|\pi_{D}(p) \pi_{D}(q)\right| & \leq\left|\pi_{D}(p) \pi_{D}(\hat{p})\right|+\left|\pi_{D}(\hat{p}) \pi_{D}(\hat{q})\right|+\left|\pi_{D}(\hat{q}) \pi_{D}(q)\right| \\
& \leq \varepsilon+|\hat{p} \hat{q}|+\varepsilon \\
& \leq 2 \varepsilon+2 \delta+|p q|
\end{aligned}
$$

By choosing $\varepsilon$ and hence $\delta$ arbitrarily small, the result follows.

10.7 Corollary The souls form an equidistant fibration of $M$. All dual fibers are isometric and form convex subsets of $M$.

Proof Let $S_{1}, S_{2}$ be souls and $p \in S_{1}, q \in S_{2}$ such that $\left|S_{1} S_{2}\right|=|p q|$. By Proposition 10.6, the shortest path $p q$ can be transported via projection along souls into any dual fiber $D$ and satisfies length $\left(\pi_{D}(p q)\right) \leq|p q|$. Thus, equality follows, which proves the first statement and therewith also the second statement.

\section{The Splitting Theorem}

Now the proof of the Splitting Theorem and its corollaries can be completed. 
Proof of Theorem 1.2 for $k=\ell$ We pick arbitrarily a soul $S$ and a dual fiber $D$. Since the set of souls is equidistant and so is the set of dual fibers, $M$ is isometric to the product $S \times D$ equipped with the product metric. Indeed, in $M$ the Pythagorean Theorem holds up to an error of order $o(h)$ if $h$ is some side length of a rectangular triangle. Since souls and dual fibers intersect perpendicularly, we obtain the canonical isometry $\pi_{S} \times \pi_{D}: M \rightarrow S \times D$, where $\pi_{S}, \pi_{D}$ are the canonical projections.

In order to complete the proof in full generality, we keep the notation as fixed in Section 6, but allow $k \leq \ell$.

Proof of Theorem 1.2 in general So far, the splitting is proved if $F_{1}, \ldots, F_{k+1}$ is a stratification of $\partial M$. In this case, souls are Alexandrov spaces without boundary, which is essential for the proof of Proposition 7.4. Assume now that $\partial M$ contains additional boundary strata $F_{k+2}, \ldots, F_{\ell+1}$. This does not affect the results till Proposition 5.6. Also this one carries over with minor changes, namely, $A_{i} \cap F_{k+2}, \ldots, A_{i} \cap F_{\ell+1}$ form additional boundary strata of the maximum set $A_{i}$. This follows according to Lemma 3.5 if we prove that $A_{i}$ is not contained in any stratum $F_{k+1}, \ldots, F_{\ell+1}$. Indeed, if we consider the space $\bar{M}$ by gluing along $F_{k+2} \cup \ldots \cup F_{\ell+1}$, we obtain that $\operatorname{dim} \bar{A}_{i}=\operatorname{dim} A_{i}$. By construction, we have that $A_{i} \subseteq F_{j}$ if, and only if, $\overline{A_{i}} \subseteq \bar{F}_{j}$ for $j \in\{1, \ldots, k+1\}$. Hence, $A_{i}$ cannot be contained in any additional boundary strata, since this would imply a smaller dimension.

The extended Proposition 5.6 allows us to assume by induction that the Splitting Theorem holds in full generality for maximum sets $A_{i}$. Therefore, we obtain a fibration of $M$ into convex sets of dimension $n-k$ like in Proposition 7.1. These sets, however, have now boundary. For simplicity, we still call them "souls" and obtain that each soul $S \subseteq M$ has boundary stratification $S \cap F_{k+2}, \ldots, S \cap F_{\ell+1}$. Now we can consider again the doubling $\bar{M}$ from above and apply the Splitting Theorem for $k=\ell$. We obtain the product structure $\bar{M}=\bar{S} \times D$. Here, $\bar{S}$ is exactly the doubling of $S$, since the boundary strata of $S$ are induced by $F_{k+2}, \ldots, F_{\ell+1}$. Therefore, $M$ inherits the product structure and is isometric to $S \times D$. This shows that the Splitting Theorem also holds in full generality.

Proof of Corollary 1.3 In the Alexandrov category, a point has positive curvature if it possesses a neighborhood $U$ such that each triangle in $U$ fulfills the comparison condition with a comparison space of positive curvature. In particular, there is no triangle in $U$ for which the Pythagorean Theorem holds. If $M$ is a (nontrivial) metric product, however, there are Pythagorean triangles at each point.

Proof of Corollary 1.5 The result follows immediately from Theorem 1.2 and Theorem 1.4. 
Proof of Corollary 1.6 Let $M$ be a compact nonnegatively curved Alexandrov space of dimension $n$. Assume that $\partial M$ consists of more than $n+1$ boundary strata. By Theorem 1.4, any intersection of $n+1$ boundary strata is empty and there must be an intersection of $n$ boundary strata which is also empty. Hence, Theorem 1.2 implies that $M$ is a nontrivial metric product, that is, the $S$-factor has dimension at least 1 .

Proof of Corollary 1.7 The result follows from Corollary 1.5 and Theorem 1.2. Note also that $M$ cannot possess more than $n+1$ boundary strata. It is easy to prove this directly, but it immediately follows from Corollary 1.6 and Corollary 1.3.

Corollary 1.8 follows by iterated use of Theorem 1.2. As mentioned, Perelman had proved the results directly, so we only sketch our proof here for completeness. For more details see the author's thesis [31, Theorem 4.5].

Sketch of proof for Corollary 1.8 Let $M$ be a compact nonnegatively curved Alexandrov space of dimension $n$. Let $F_{1}, \ldots, F_{\ell}$ be a stratification of $\partial M$. For any $p \in M \backslash \partial M$ we have that $\left|\uparrow F_{i} \uparrow F_{p}\right| \geq \frac{\pi}{2}$ for all $i \neq j$. This implies that $\ell \leq 2 n$. Now assume that equality holds. It is easy to see that all points in $M \backslash \partial M$ are regular and shortest paths to any $F_{i}$ are unique. Pick some $p \in M \backslash \partial M$ and assume, by renumbering, that $\left|\uparrow p_{p} \uparrow F_{p}\right|=\pi$. It follows from semicontinuity of angles that such points form an open set. This in turn implies that all points $p \in M \backslash \partial M$ fulfill that $\left|\uparrow F_{1} \uparrow_{p} F_{2}\right|=\pi$. Hence, $F_{1} \cap F_{2}=\varnothing$, and Theorem 1.2 can be applied. The $D$-factor is an interval, while the $S$-factor has dimension $n-1$ and contains $2 n-2$ boundary strata. Iteration proves the statement.

Sketch of proof for Corollary 1.9 We consider the rays corresponding to the $m+2$ points $p_{1}, \ldots, p_{m+2}$ in the ideal boundary at distance $>\frac{\pi}{2}$ from each other. Further, we denote by $b_{1}, \ldots, b_{m+2}$ the Busemann functions corresponding to the rays. Let $\left(a_{i}\right)$ be a monotonely increasing diverging sequence such that the intersections of sublevel sets $M_{i}:=b_{1}^{-1}\left(\left[0, a_{i}\right]\right) \cap \ldots \cap b_{m+2}^{-1}\left(\left[0, a_{i}\right]\right)$ are non-collapsed for all $i \in \mathbb{N}$. Then each $M_{i}$ is a compact nonnegatively curved Alexandrov space with $m+2$ boundary strata. Indeed, if it is not compact, we find a ray and therewith a point $q$ in the ideal boundary distinct from $p_{1}, \ldots, p_{m+2}$. Since the distance function to each boundary stratum is concave, we conclude that $d\left(q, p_{\ell}\right) \geq \frac{\pi}{2}$ for $\ell=1, \ldots, m+2$, which is impossible.

For each $i$ we denote the boundary strata of $M_{i}$ by $F_{i}^{1}, \ldots, F_{i}^{m+2}$. Now Theorem 1.2 implies the following. If there exists a collection of strata $F_{i}^{n_{1}}, \ldots, F_{1}^{n_{k+1}}$ with minimal $k$ such that $F_{i}^{n_{1}} \cap \ldots \cap F_{1}^{n_{k}}=\varnothing$ holds for infinitely many indices $i$, then $M$ splits off its soul of codimension $k$. For $k<m+1$ this would imply that the 
$(m+1)$-dimensional cone over the ideal boundary also splits. This contradicts the existence of $p_{1}, \ldots, p_{m+2}$. On the other hand, if we show that for $k=m+1$ the intersection from above is indeed empty for infinitely many indices $i$, the Corollary is proved. We assume, by way of contradiction, that $F_{i}^{1} \cap \ldots \cap F_{i}^{m+2}$ is nonempty for almost all indices $i$. If we rescale the spaces $M_{i}$ keeping the diameter constant, we see that the asymptotic cone of this sequence coincides with the asymptotic cone of $M$ cut down to the intersection of sublevel sets of the Busemann functions. In particular, we get an intersection of $m+2$ boundary strata in a space of dimension $m+1$, contradiction.

\section{References}

[1] S B Alexander, R L Bishop, A cone splitting theorem for Alexandrov spaces, Pacific J. Math. 218 (2005) 1-15 MR2224586

[2] D Burago, Y Burago, S Ivanov, A course in metric geometry, Graduate Studies in Mathematics 33, American Mathematical Society (2001) MR1835418

[3] Y Burago, M Gromov, G Perelman, A. D. Aleksandrov spaces with curvatures bounded below, Uspekhi Mat. Nauk 47 (1992) 3-51, 222 MR1185284

[4] J Cheeger, D Gromoll, The splitting theorem for manifolds of nonnegative Ricci curvature, J. Differential Geometry 6 (1971/72) 119-128 MR0303460

[5] J Cheeger, D Gromoll, On the structure of complete manifolds of nonnegative curvature, Ann. of Math. 96 (1972) 413-443 MR0309010

[6] K Grove, P Petersen, A radius sphere theorem, Invent. Math. 112 (1993) 577-583 MR1218324

[7] V Kapovitch, Perelman's stability theorem, from: "Surveys in differential geometry Vol XI”, Surv. Differ. Geom. 11, Int. Press, Somerville, MA (2007) 103-136 MR2408265

[8] A Lytchak, Allgemeine Theorie der Submetrien und verwandte mathematische Probleme, Bonner Mathematische Schriften 347, Universität Bonn Mathematisches Institut (2002) MR1938523 Dissertation, Rheinische Friedrich-Wilhelms-Universität Bonn, Bonn, 2001

[9] A Lytchak, Open map theorem for metric spaces, Algebra i Analiz 17 (2005) 139-159 MR2167848

[10] Y Mashiko, A splitting theorem for Alexandrov spaces, Pacific J. Math. 204 (2002) 445-458 MR1907900

[11] S J Mendonça, The asymptotic behavior of the set of rays, Comment. Math. Helv. 72 (1997) 331-348 MR1476053

[12] A D Milka, Metric structure of a certain class of spaces that contain straight lines, Ukrain. Geometr. Sb. Vyp. 4 (1967) 43-48 MR0256327 
[13] A Mitsuishi, A splitting theorem for infinite dimensional Alexandrov spaces with nonnegative curvature and its applications, Geom. Dedicata 144 (2010) 101-114 MR2580420

[14] Y Otsu, Differential geometric aspects of Alexandrov spaces, from: "Comparison geometry (Berkeley, CA, 1993-94)”, Math. Sci. Res. Inst. Publ. 30, Cambridge Univ. Press (1997) 135-148 MR1452870

[15] Y Otsu, T Shioya, The Riemannian structure of Alexandrov spaces, J. Differential Geom. 39 (1994) 629-658 MR1274133

[16] G Perelman, Alexandrov's spaces with curvatures bounded from below II, preprint (1991) Available at http://www.math.psu.edu/petrunin/papers/ papers.html

[17] G Perelman, Elements of Morse theory on Aleksandrov spaces, Algebra i Analiz 5 (1993) 232-241 MR1220498

[18] G Perelman, Proof of the soul conjecture of Cheeger and Gromoll, J. Differential Geom. 40 (1994) 209-212 MR1285534

[19] G Perelman, Collapsing with no proper extremal subsets, from: "Comparison geometry (Berkeley, CA, 1993-94)”, Math. Sci. Res. Inst. Publ. 30, Cambridge Univ. Press (1997) 149-155 MR1452871

[20] G Perelman, A M Petrunin, Extremal subsets in Aleksandrov spaces and the generalized Liberman theorem, Algebra i Analiz 5 (1993) 242-256 MR1220499

[21] G Perelman, A Petrunin, Quasigeodesics and gradient curves in Alexandrov spaces, preprint (1995) Available at http://www.math.psu.edu/petrunin/papers/ papers.html

[22] A Petrunin, Applications of quasigeodesics and gradient curves, from: "Comparison geometry (Berkeley, CA, 1993-94)”, Math. Sci. Res. Inst. Publ. 30, Cambridge Univ. Press (1997) 203-219 MR1452875

[23] A Petrunin, Parallel transportation for Alexandrov space with curvature bounded below, Geom. Funct. Anal. 8 (1998) 123-148 MR1601854

[24] A Petrunin, Semiconcave functions in Alexandrov's geometry, from: "Surveys in differential geometry Vol XI", Surv. Differ. Geom. 11, Int. Press, Somerville, MA (2007) 137-201 MR2408266

[25] V A Sharafutdinov, Convex sets in a manifold of nonnegative curvature, Mat. Zametki 26 (1979) 129-136, 159 MR553571 English translation in Math. Notes 26 (1979) $556-560$

[26] T Shioya, Splitting theorems for non-negatively curved open manifolds with large ideal boundary, Math. Z. 212 (1993) 223-238 MR1202809

[27] M Strake, A splitting theorem for open nonnegatively curved manifolds, Manuscripta Math. 61 (1988) 315-325 MR949821 
[28] V A Toponogov, Riemannian spaces which contain straight lines, Amer. Math. Soc. Transl. Ser. 237 (1964) 287-290

[29] G Walschap, A splitting theorem for 4-dimensional manifolds of nonnegative curvature, Proc. Amer. Math. Soc. 104 (1988) 265-268 MR958080

[30] B Wilking, Positively curved manifolds with symmetry, Ann. of Math. 163 (2006) 607-668 MR2199227

[31] A Wörner, Boundary strata of nonnegatively curved Alexandrov spaces and a splitting theorem, $\mathrm{PhD}$ thesis, WWU Münster (2010) Available at http:// miami . uni-muenster .de/servlets/DocumentServlet?id=5349

[32] J-W Yim, Space of souls in a complete open manifold of nonnegative curvature, J. Differential Geom. 32 (1990) 429-455 MR1072913

Charlottenstraße 29, 72070 Tübingen, Germany

a.woerner@arcor.de

Proposed: Dmitri Burago

Seconded: Tobias H. Colding, Leonid Polterovich
Received: 11 August 2011

Revised: 18 August 2012 